\title{
OPTIMAL OPERATOR PRECONDITIONING FOR GALERKIN BOUNDARY ELEMENT METHODS ON 3D SCREENS
}

\author{
RALF HIPTMAIR*, CARLOS JEREZ-HANCKES ${ }^{\dagger}$, AND CAROLINA URZÚA-TORRES $\ddagger$
}

\begin{abstract}
We consider first-kind weakly singular and hypersingular boundary integral operators for the Laplacian on screens in $\mathbb{R}^{3}$ and their Galerkin discretization by means of low-order piecewise polynomial boundary elements. For the resulting linear systems of equations we propose novel Calderón-type preconditioners based on (i) new boundary integral operators, which provide the exact inverses of the weakly singular and hypersingular operators on flat disks, and (ii) stable duality pairings relying on dual meshes. On screens obtained as images of the unit disk under bi-Lipschitz transformations, this approach achieves condition numbers uniformly bounded in the meshwidth even on locally refined meshes. Comprehensive numerical tests also confirm its excellent pre-asymptotic performance.
\end{abstract}

Key words. Operator preconditioning, boundary integral operators, boundary element methods, screens

AMS subject classifications. 65R20, 65F $35,65 \mathrm{~N} 22,65 \mathrm{~N} 38$

1. Galerkin Boundary Element Method (BEM) for exterior screen problems. Throughout, let $\Gamma \subset \mathbb{R}^{3}$ stand for an orientable, piecewise smooth, Lipschitz manifold, also called a screen in literature.

1.1. Boundary Value Problems (BVPs). We consider the following exterior Dirichlet and Neumann BVPs in the complement $\Omega:=\mathbb{R}^{3} \backslash \bar{\Gamma}$ of $\Gamma$, see [22, Section 3.5.3],

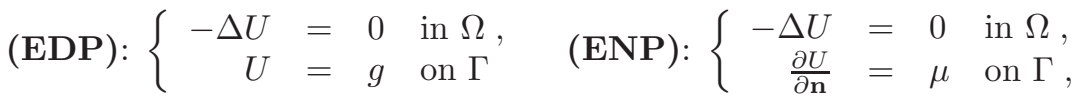

both to be supplemented with decay conditions $U(\mathbf{x})=\mathcal{O}\left(\left\|\mathbf{x}^{-1}\right\|\right)$ as $\|\mathbf{x}\| \rightarrow \infty$. They model several physical phenomena: the potential distribution outside thin metal sheets in electrostatics; the temperature distribution in the presence of thin inclusions; cracks in stationary heat conduction; fractures in linear elasticity, to name a few examples.

\section{2. (Variational) Boundary Integral Equations (BIEs).}

1.2.1. Weakly Singular Boundary Integral Equation. For (EDP) assume $g \in H^{1 / 2}(\Gamma) 1$. Then the jump across $\Gamma$ of the Neumann trace of its solution, denoted by $\sigma \in \widetilde{H}^{-1 / 2}(\Gamma)$, is the solution of the weakly singular BIE:

$$
(\mathrm{V} \sigma)(\mathbf{x}):=\frac{1}{4 \pi} \int_{\Gamma} \frac{\sigma(\mathbf{y})}{\|\mathbf{x}-\mathbf{y}\|} d \Gamma(\mathbf{y})=g(\mathbf{x}), \quad \mathbf{x} \in \Gamma .
$$

*Seminar for Applied Mathematics, ETH Zurich, Raemistrasse 101, 8092 Zurich, Switzerland. ralf.hiptmair@sam.math.ethz.ch.

†School of Engineering, Pontificia Universidad Católica de Chile, Av. Vicuña Mackenna 4860, Macul, Santiago, Chile. cjerez@ing.puc.cl. Prof. Jerez-Hanckes' work was partially funded by Fondecyt Regular 1171491 and Chile CORFO Engineering 2030 program through grant OPEN-UC 201603.

${ }_{\ddagger}^{\ddagger}$ Mathematical Institute, University of Oxford, UK, carolina.urzuatorres@maths.ox.ac.uk The work of C. Urzúa-Torres was partly funded by ETH Zurich through ETHIRA grant ETH-04 13-2.

${ }^{1}$ For notations and theory of Sobolev (trace) spaces we refer to [22, Section 2.4] and [18] Chapter 3] 
Here, $d \Gamma(\mathbf{x})$ designates the surface measure at $\mathbf{x} \in \Gamma$. $\mathbf{V}$ denotes the weakly singular Boundary Integral Operator (BIO). Writing $\langle\cdot, \cdot\rangle_{\Gamma}$ for the $L^{2}(\Gamma)$-inner product and induced duality pairings, let us define the bilinear form:

$$
\mathrm{av}, \Gamma(\sigma, \psi):=\langle\mathrm{V} \sigma, \psi\rangle_{\Gamma}=\frac{1}{4 \pi} \int_{\Gamma} \int_{\Gamma} \frac{\sigma(\mathbf{y}) \psi(\mathbf{x})}{\|\mathbf{x}-\mathbf{y}\|} d \Gamma(\mathbf{y}) d \Gamma(\mathbf{x}),
$$

for all $\sigma, \psi \in \widetilde{H}^{-1 / 2}(\Gamma)$. Then, the symmetric variational formulation for (1.2) is 22, Sect. 3.5.3]: seek $\sigma \in \widetilde{H}^{-1 / 2}(\Gamma)$ such that

$$
\mathrm{av}, \Gamma(\sigma, \psi)=\langle g, \psi\rangle_{\Gamma}, \quad \forall \psi \in \widetilde{H}^{-1 / 2}(\Gamma) .
$$

1.2.2. Hypersingular Integral Equation. From [22, Sect. 3.5.3] we learn that in the case of (ENP) with data $\mu \in H^{-1 / 2}(\Gamma)$, the jump of the Dirichlet trace of $U$ is available as the solution $u \in \widetilde{H}^{1 / 2}(\Gamma)$ of the integral equation 2

$$
(\mathbf{W} u)(\mathbf{x}):=-\frac{1}{4 \pi} f_{\Gamma} u(\mathbf{y}) \frac{\partial^{2}}{\partial n_{x} \partial n_{y}} \frac{1}{\|\mathbf{x}-\mathbf{y}\|} d \Gamma(\mathbf{y})=\mu(\mathbf{x}), \quad \mathbf{x} \in \Gamma .
$$

The statement of the weak form of (1.5) relies on the vectorial surface curl operator [24. p. 133]. For a continuously differentiable function $v$ on $\Gamma$ with an appropriate smooth extension $\tilde{v}$ to a three-dimensional neighborhood of $\Gamma$, one defines

$$
\operatorname{curl}_{\Gamma, \mathbf{x}} v(\mathbf{x}):=\mathbf{n}(\mathbf{x}) \times \nabla \tilde{v}(\mathbf{x}), \quad \mathbf{x} \in \Gamma,
$$

with $\mathbf{n}(\mathbf{x})$ being a normal vector to $\Gamma$, and $\nabla$ denoting the standard gradient. Then, a symmetric variational problem for (1.5) is given by: seek $u \in \widetilde{H}^{1 / 2}(\Gamma)$ such that

$$
\langle\mathrm{W} u, v\rangle_{\Gamma}:=\frac{1}{4 \pi} \int_{\Gamma} \int_{\Gamma} \frac{\operatorname{curl}_{\Gamma, \mathbf{y}} u(\mathbf{y}) \cdot \operatorname{curl}_{\Gamma, \mathbf{x}} v(\mathbf{x})}{\|\mathbf{x}-\mathbf{y}\|} d \Gamma(\mathbf{x}) d \Gamma(\mathbf{y})=\langle\mu, v\rangle_{\Gamma},
$$

for all $v \in \widetilde{H}^{1 / 2}(\Gamma)$. We write aw, for the bilinear form on the left-hand side of (1.6).

Existence and uniqueness of solutions for problems (1.4) and (1.6) was proved by Stephan in [26, Thm. 2.7]. Moreover, for a screen $\Gamma$, the bilinear forms in (1.4) and (1.6) are continuous and elliptic in $\widetilde{H}^{-1 / 2}(\Gamma)$ and $\widetilde{H}^{1 / 2}(\Gamma)$, respectively ( $c f$. [22, Thm. 3.5.9]). One can show that in these cases and for sufficiently smooth screens $\Gamma$, when approaching the edges $\partial \Gamma$, solutions of (1.5) decay like the square-root of the distance to $\partial \Gamma$ [7, whereas those of (1.2) blow-up like the reciprocal square root.

1.3. Galerkin BEM Discretization. In the spirit of [22, Definition 4.1.4] we equip the screen $\Gamma$ with a regular triangular surface mesh $\Gamma_{h}=\{\tau\}$, with index $h>0$, whose elements $\tau$ are supposed to be diffeomorphic images of a flat reference triangle. For Galerkin discretization of the variational equations (1.4) and (1.6) we rely on standard $\Gamma_{h}$-piecewise (mapped) polynomial boundary element spaces as introduced in [22, Sections 4.1.3 \& 4.1.7]:

- (EDP), (1.4): We use the space

$$
\mathcal{S}^{-1,0}\left(\Gamma_{h}\right):=\text { "space of piecewise constant functions on } \Gamma_{h} \text { " } \subset \widetilde{H}^{-1 / 2}(\Gamma) \text {. }
$$

Its dimension agrees with the number of elements of $\Gamma_{h}$ and the characteristic functions of the mesh elements provide the customary basis $\left\{\varphi_{\tau}\right\}_{\tau \in \Gamma_{h}}$.

\footnotetext{
${ }^{2}$ Finite part integrals with distributional meaning as in [18 Chapter 5] are labeled with a dash as in $f$.
} 
- (ENP), (1.6): We employ the boundary element space

$$
\begin{aligned}
\mathcal{S}_{0}^{0,1}\left(\Gamma_{h}\right):= & \text { "space of continuous piecewise linear functions on } \Gamma_{h}, \\
& \text { satisfying zero boundary conditions on } \partial \Gamma " \subset \widetilde{H}^{1 / 2}(\Gamma) .
\end{aligned}
$$

Its dimension agrees with the number of interior nodes of $\Gamma_{h}$ and we use the standard "nodal" basis comprised of (mapped) "tent functions" $\varphi_{v}$, with $v$ from the set of vertices of $\Gamma_{h}$, and $\varphi_{v}(v)=1$.

In both cases, we arrive at dense linear systems of equations featuring symmetric positive definite coefficient matrices. Naturally, since we deal with first-kind boundary integral operators of order \pm 1 and use merely $L^{2}(\Gamma)$-stable bases, the spectral condition numbers of the Galerkin matrices can be expected to increase like $O\left(h_{\mathrm{min}}^{-1}\right)$ for shrinking minimal element size $h_{\min } \rightarrow 0$ of the mesh (cf. [22, Section 4.5] for a detailed exposition).

In this investigation we adopt an asymptotic perspective, studying the behavior of algorithms for "meshwidth $\rightarrow$ zero". To that end, we rely on a family of meshes $\left\{\Gamma_{h}\right\}_{h \in \mathbb{H}}$ of $\Gamma$, indexed by $h$ from an index set $\mathbb{H} \subset \mathbb{R}^{+}$with accumulation point zero; $h$ should be read as the meshwidth of $\Gamma_{h}$. Appealing to [22, Definition 4.1.12] we assume limited distortion of element shapes:

Assumption 1.1. The family $\left\{\Gamma_{h}\right\}_{h \in \mathbb{H}}$ of meshes is assumed to be uniformly shape-regular, i.e. the shape-regularity constants of the $\Gamma_{h}$ are $h$-uniformly bounded.

REMARK 1. We must not assume global quasi-uniformity of $\left\{\Gamma_{h}\right\}_{h \in \mathbb{H}}$ according to [22, Eq. (4.1.9)] as it rules out locally refined meshes, because the singular behavior of generic solutions of (1.4) and (1.6) at $\partial \Gamma$ suggests a mesh refinement towards the boundary of the screen.

2. Operator Preconditioning. Operator preconditioning is a general and powerful policy for devising mesh-robust preconditioners for Galerkin matrices.

2.1. Abstract Theory. The best way to introduce this preconditioning approach is through the abstract framework first presented in [5. Prop. 1.2.1] and elaborated in [10], see also the Introduction of [11, from where we cite Theorem 1.1:

Theorem 2.1 (Theorem 1.1 [1]). Let $X, Y$ be reflexive Banach spaces, $X_{h}:=$ $\operatorname{span}\left\{\varphi_{i}\right\}_{i=0}^{N} \subset X, Y_{h}:=\operatorname{span}\left\{\phi_{j}\right\}_{j=0}^{M} \subset Y$ finite-dimensional subspaces with bases $\left\{\varphi_{i}\right\}_{i=0}^{N}$ and $\left\{\phi_{j}\right\}_{j=0}^{M}$. Further, let $\mathrm{a} \in L(X \times X, \mathbb{C})$ and $\mathrm{b} \in L(Y \times Y, \mathbb{C})$ be continuous sesquilinear forms (with norms $\|\mathrm{a}\|$ and $\|\mathrm{b}\|$, resp.), each satisfying discrete inf-sup conditions with constants $c_{A}, c_{B}>0$ on $X_{h}$ and $Y_{h}$, respectively. If there is a continuous sesquilinear form $\mathrm{t} \in L(X \times Y, \mathbb{C})$ that also satisfies discrete inf-sup conditions on $X_{h} \times Y_{h}$ with constant $c_{T}>0$, then the associated Galerkin matrices:

$$
\mathbf{A}_{h}:=\left(\mathrm{a}\left(\varphi_{i}, \varphi_{j}\right)\right)_{i, j=1}^{N}, \mathbf{B}_{h}:=\left(\mathrm{b}\left(\phi_{i}, \phi_{j}\right)\right)_{i, j=1}^{M}, \mathbf{T}_{h}:=\left(\mathrm{t}\left(\varphi_{i}, \phi_{j}\right)\right)_{i, j=1}^{N, M},
$$

satisfy

$$
\kappa\left(\mathbf{T}_{h}^{-1} \mathbf{B}_{h} \mathbf{T}_{h}^{-H} \mathbf{A}_{h}\right) \leq \frac{\|\mathrm{a}\|\|\mathrm{b}\|\|\mathrm{t}\|^{2}}{c_{A} c_{B} c_{T}^{2}},
$$

if $N:=\operatorname{dim} X_{h}=\operatorname{dim} Y_{h}=: M$. Here $\kappa$ designates the spectral condition number. 
Of course, in this work the bilinear forms av, $\Gamma$ and aw, $\Gamma$ will play the role of a with $X=\widetilde{H}^{-1 / 2}(\Gamma) / X_{h}=\mathcal{S}^{-1,0}\left(\Gamma_{h}\right)$ and $X=\widetilde{H}^{1 / 2}(\Gamma) / X_{h}=\mathcal{S}_{0}^{0,1}\left(\Gamma_{h}\right)$, respectively. The presence of the inverses $\mathbf{T}_{h}^{-1}$ and $\mathbf{T}_{h}^{-H}$ in (2.2) hints that a practical preconditioner $\mathbf{T}_{h}^{-1} \mathbf{B}_{h} \mathbf{T}_{h}^{-H}$ can be obtained only if $\mathbf{T}_{h}$ is sparse. Since we use localized basis function, this is guaranteed if we stipulate that

$$
\mathrm{t} \in L\left(X_{h} \times Y_{h}, \mathbb{R}\right) \text { is the } L^{2}(\Gamma) \text {-inner product: } \mathrm{t}(\varphi, \psi):=\langle\varphi, \psi\rangle_{\Gamma} .
$$

Accepting this and demanding that all constants in (2.2) be independent of $h \in \mathbb{H}$ for the given family $\left\{\Gamma_{h}\right\}_{h \in \mathbb{H}}$ of finer and finer meshes, we conclude that $X$ and $Y$ must be in $L^{2}(\Gamma)$-duality.

2.2. Calderón preconditioning for Galerkin BEM on closed surfaces. If $\Gamma$ is a Lipschitz surface without boundary, an $L^{2}(\Gamma)$-dual pair of spaces is available immediately, namely the trace spaces $H^{1 / 2}(\Gamma)$ and $H^{-1 / 2}(\Gamma)$ [18, Theorem 3.30], which agree with $\widetilde{H}^{1 / 2}(\Gamma)$ and $\widetilde{H}^{-1 / 2}(\Gamma)$, respectively, in this geometrical setting. Then, on closed surfaces we can choose $a=a v, \Gamma$ and $b=a w_{, \Gamma}$ and vice versa! Of course, the low-dimensional kernel of the hypersingular operator has to be suppressed by suitable regularization of $\mathrm{aw}_{\mathrm{w}, \Gamma}$ 24, p. 176]. This choice has been dubbed opposite order preconditioning. The idea comes from canceling the symbol order of the underlying pseudo-differential operator. It is also known as Calderón preconditioning in light of the famous Calderón identities [22, (3.125b)] that reveal that $\mathrm{VW}$ and $\mathrm{W} \mathrm{V}$ are bounded operators in $L^{2}(\Gamma)$ on closed surfaces. Calderón preconditioning was pioneered by O. Steinbach and W. Wendland in [25], then pursued by S. Christiansen and J.-C. Nédélec [6], and later extended to electromagnetic BIEs on closed surfaces by A. Buffa and S. Christiansen in [4. We point out that ensuring an $h$-uniform inf-sup condition for $\mathrm{t}(\cdot, \cdot)$ on boundary element spaces entails elaborate constructions. We are coming back to this in Section 4

2.3. The screen challenge. Unfortunately, the spaces $\widetilde{H}^{-1 / 2}(\Gamma)$ and $\widetilde{H}^{1 / 2}(\Gamma)$ fail to be $L^{2}(\Gamma)$-dual on screens, that is, whenever $\partial \Gamma \neq \emptyset$. In this case we find the $L^{2}(\Gamma)$-dualities [16]:

$$
\left(\widetilde{H}^{-1 / 2}(\Gamma)\right)^{\prime} \equiv H^{1 / 2}(\Gamma) \quad, \quad\left(\widetilde{H}^{1 / 2}(\Gamma)\right)^{\prime} \equiv H^{-1 / 2}(\Gamma) !
$$

In addition, $\widetilde{H}^{ \pm 1 / 2}(\Gamma)$ are not closed subspaces of $H^{ \pm 1 / 2}(\Gamma)$; the norms of the former spaces are strictly stronger than those of the latter.

If we chose to ignore this mismatch and opted for opposite order preconditioning exactly as for closed surfaces we would incur a logarithmic growth $O(\log h)$ of the condition number. This was first proved in [19] for open curves in $2 \mathrm{D}$, and then also for open three-dimensional surfaces in [5, Prop. 1.3.5].

An improvement on the preconditioning was achieved by Bruno and Lintner [2, 3, who introduced weights in the kernels of the BIOs. Such weights depend on the distance to $\partial \Gamma$ and incorporate information on the singular behavior of the solution as in augmented schemes [27. The same heuristics underlies the preconditioning operators proposed by J.-C. Nédélec and P. Ramacciotti in [20. In these works better overall performance of operator preconditioning in numerical experiments is achieved, but rigorous numerical analysis is missing.

At any rate, in order to perform operator preconditioning with "optimal" $h$ uniform bound on the condition numbers, we cannot rely on the available BIOs to supply suitable bilinear forms $\mathrm{b}$. New operators $\overline{\mathrm{V}}$ and $\overline{\mathrm{W}}$ have to be designed that map continuously $\overline{\mathrm{V}}: H^{-1 / 2} \rightarrow \widetilde{H}^{1 / 2}(\Gamma)$ and $\overline{\mathrm{W}}: H^{1 / 2} \rightarrow \widetilde{H}^{-1 / 2}(\Gamma)$ and, hence, 
give rise to continuous bilinear forms on $H^{-1 / 2}(\Gamma)$ and $H^{1 / 2}(\Gamma)$, respectively. This was first accomplished in 2D for line segments in [16]. There the authors found exact (variational) inverses for the $2 \mathrm{D}$ counterparts of $\mathrm{V}$ and $\mathrm{W}$ ! These inverses are introduced as modified weakly singular and hypersingular operators, and one observes that they also incorporate the distance from $\partial \Gamma$ in their kernels. They were successfully used for optimal operator preconditioning in 11]. 3D counterparts for $\bar{V}$ and $\bar{W}$ were subsequently constructed by P. Ramacciotti in [21, Sect. 2.7] in the form of series representations, which, however, turned out to be useless for the design of preconditioners.

2.4. Outline, main result, and novelty. The success of [16, 11] for open curves prompted the research underlying this manuscript. Yet, [16] heavily relies on spectral decompositions using Chebychev polynomials. These techniques are specifically tailored to line segments and smooth arcs and do not carry over to screens. A breakthrough could be achieved thanks to a key discovery, reported in 13, namely that of exact (variational) inverses of both $\mathrm{V}$ and $\mathrm{W}$ on flat disks (see the next section for a summary). This made it possible to harness Steinbach's 23 comprehensive theory of stable discrete $L^{2}$-duality pairings, which is reviewed and adapted in Section 4 . Bi-Lipschitz pullbacks to disks permit us to apply the new method for operator preconditioning to a wide class of rather general screens, see Section 5 . Thus, in this work we have designed the

first rigorously $h$-uniformly performing (also called "asymptotically optimal", "scalable") operator preconditioning algorithm for piecewise polynomial Galerkin BEM for the weakly singular and hypersingular BIE on screens that are bi-Lipschitz images of flat disks.

Rigorous statements of the main theoretical results are given in Corollaries 4.3 and [5.1. In Section 5.3 we briefly remark on an alternative "heuristic" way to design preconditioning operators. Numerical experiments reported in the final section confirm that the method achieves condition numbers that are not only bounded independently of $h$, but fairly close to one.

3. Exact inverse BIOs over the disk. For the sake of completeness we summarize the results of [13] in this section. We write $\mathbb{D}_{a}$ for the flat circular disk with radius $a>0$, defined as $\mathbb{D}_{a}:=\left\{\mathbf{x} \in \mathbb{R}^{3}: x_{3}=0\right.$ and $\left.\|\mathbf{x}\|<a\right\}$.

3.1. Modified Weakly Singular Integral Operator. We define the modified weakly singular operator as in [13, Eq. (3.1)] 3 4

$$
(\overline{\mathrm{V}} v)(\mathbf{x}):=\frac{2}{\pi^{2}} \int_{\mathbb{D}_{a}} v(\mathbf{y}) \frac{S_{a}(\mathbf{x}, \mathbf{y})}{\|\mathbf{x}-\mathbf{y}\|} d \mathbb{D}_{a}(\mathbf{y}), \quad \mathbf{x} \in \mathbb{D}_{a},
$$

with a bounded modulation function $S_{a} \in L^{\infty}\left(\mathbb{D}_{a} \times \mathbb{D}_{a}\right)$ given by

$$
S_{a}(\mathbf{x}, \mathbf{y}):=\tan ^{-1}\left(\frac{\sqrt{a^{2}-\|\mathbf{x}\|^{2}} \sqrt{a^{2}-\|\mathbf{y}\|^{2}}}{a\|\mathbf{x}-\mathbf{y}\|}\right), \quad \mathbf{x} \neq \mathbf{y} .
$$

Note that $\overline{\mathrm{V}}$ features a weakly singular integrable kernel with leading singularity of $O\left(\|\mathbf{x}-\mathbf{y}\|^{-1}\right)$, which allows the generation of Galerkin BEM matrices in exactly the same way as for the bilinear form $\mathrm{av}, \Gamma$, see [22, Chapter 5].

\footnotetext{
${ }^{3}$ Compared to [13 Eq. (3.1)] a sign change is applied to match 1.5.

${ }^{4}$ The measure $d \mathbb{D}_{a}(\mathbf{x})$ denotes the surface element in terms of $\mathbf{x} \in \mathbb{D}_{a}$.
} 


\begin{tabular}{|c|c|c|c|c|c|}
\hline BVP & $\mathrm{a}$ & $\mathrm{b}$ & $X$ & $Y$ & $X_{h}$ \\
\hline \hline (EDP) & $\mathrm{a}_{\mathrm{v}, \mathbb{D}_{a}}, c f .(1.3)$ & $\mathrm{b}_{\overline{\mathrm{W}}, a}, c f .(3.8 \mathrm{~b}$ & $\widetilde{H}^{-1 / 2}(\Gamma)$ & $H^{1 / 2}(\Gamma)$ & $\mathcal{S}^{-1,0}\left(\Gamma_{h}\right)$ \\
\hline (ENP) & $\mathrm{a}_{\mathrm{W}, \mathbb{D}_{a}}, c f .(1.6)$ & $\mathrm{b}_{\overline{\mathrm{V}}, a}, c f .(3.8 \mathrm{a})$ & $\widetilde{H}^{1 / 2}(\Gamma)$ & $H^{-1 / 2}(\Gamma)$ & $\mathcal{S}_{0}^{0,1}\left(\Gamma_{h}\right)$ \\
\hline
\end{tabular}

Table 3.2.1: Summary of operator preconditioning strategy for variational boundary integral equations on $\Gamma=\mathbb{D}_{a}$. In both cases the bilinear form $\mathrm{t}$ is the $L^{2}$-duality product between the spaces $X$ and $Y$.

3.2. Modified Hypersingular Integral Operator. We define the modified hypersingular operator $\overline{\mathrm{W}}$ as [13, Eq. (3.3),(3.4)]

$$
(\overline{\mathrm{W}} g)(\mathbf{x}):=-\frac{2}{\pi^{2}} f_{\mathbb{D}_{a}} g(\mathbf{y}) K_{\overline{\mathrm{W}}}(\mathbf{x}, \mathbf{y}) d \mathbb{D}_{a}(\mathbf{y}), \quad \mathbf{x} \in \mathbb{D}_{a},
$$

with

$$
K_{\overline{\mathrm{W}}}(\mathbf{x}, \mathbf{y}):=\frac{a}{\|\mathbf{x}-\mathbf{y}\|^{2} \sqrt{a^{2}-\|\mathbf{x}\|^{2}} \sqrt{a^{2}-\|\mathbf{y}\|^{2}}}+\frac{S_{a}(\mathbf{x}, \mathbf{y})}{\|\mathbf{x}-\mathbf{y}\|^{3}}, \quad \mathbf{x} \neq \mathbf{y} .
$$

The next result, analogous to (1.6), makes possible the treatment of $\overline{\mathrm{W}}$ in the context of boundary element Galerkin methods:

Proposition 3.1 ([13, Corollary 4.3]). The symmetric bilinear form associated to the modified hypersingular operator $\overline{\mathrm{W}}: H^{1 / 2}\left(\mathbb{D}_{a}\right) \rightarrow \widetilde{H}^{-1 / 2}\left(\mathbb{D}_{a}\right)$ can be written as

$$
\begin{aligned}
\langle\overline{\mathrm{W}} u, v\rangle_{\mathbb{D}_{a}}= & \frac{2}{\pi^{2}} \int_{\mathbb{D}_{a}} \int_{\mathbb{D}_{a}} \frac{S_{a}(\mathbf{x}, \mathbf{y})}{\|\mathbf{x}-\mathbf{y}\|} \operatorname{curl}_{\mathbb{D}_{a}, \mathbf{x}} u(\mathbf{x}) \cdot \operatorname{curl}_{\mathbb{D}_{a}, \mathbf{y}} v(\mathbf{y}) d \mathbb{D}_{a}(\mathbf{x}) d \mathbb{D}_{a}(\mathbf{y}) \\
& +\frac{2}{a \pi^{2}} \int_{\mathbb{D}_{a}} \int_{\mathbb{D}_{a}} \frac{u(\mathbf{x}) v(\mathbf{y})}{\omega_{a}(\mathbf{x}) \omega_{a}(\mathbf{y})} d \mathbb{D}_{a}(\mathbf{x}) d \mathbb{D}_{a}(\mathbf{y}), \quad \forall u, v \in H^{1 / 2}\left(\mathbb{D}_{a}\right),
\end{aligned}
$$

where $\omega_{a}(\mathbf{x}):=\sqrt{a^{2}-\|\mathbf{x}\|^{2}}, \mathbf{x} \in \mathbb{D}_{a}$.

Thus, Galerkin matrices for $\bar{W}$ can be obtained from those for $\bar{V}$ and a discrete surface curl operator.

Theorem 3.2 ([13, Theorem 3.1]). The following identities hold

$$
\begin{aligned}
& \overline{\mathrm{W}} \mathrm{V}=\mathrm{Id}_{\widetilde{H}^{-1 / 2}\left(\mathbb{D}_{a}\right)}, \quad \mathrm{V} \overline{\mathrm{W}}=\mathrm{Id}_{H^{1 / 2}\left(\mathbb{D}_{a}\right)}, \\
& \bar{V} \mathbf{W}=\operatorname{Id}_{\widetilde{H}^{1 / 2}\left(\mathbb{D}_{a}\right)}, \quad \mathrm{W} \bar{V}=\operatorname{Id}_{H^{-1 / 2}\left(\mathbb{D}_{a}\right)} .
\end{aligned}
$$

Recalling the properties of $\mathrm{av}_{, \Gamma}$ and $\mathrm{aw}_{\mathrm{W}, \Gamma}$, this theorem immediately confirms that the bilinear forms:

$$
\begin{array}{rlrl}
\mathrm{b}_{\overline{\mathrm{V}}, a}(\vartheta, \mu) & :=\langle\overline{\mathrm{V}} \vartheta, \mu\rangle_{\mathbb{D}_{a}}, & \vartheta, \mu \in H^{-1 / 2}\left(\mathbb{D}_{a}\right), \\
\mathrm{b}_{\overline{\mathrm{W}}_{, a}}(u, g):=\langle\overline{\mathrm{W}} u, g\rangle_{\mathbb{D}_{a}}, & u, g \in H^{1 / 2}\left(\mathbb{D}_{a}\right),
\end{array}
$$

are elliptic and continuous in $H^{-1 / 2}\left(\mathbb{D}_{a}\right)$ and $H^{1 / 2}\left(\mathbb{D}_{a}\right)$, respectively. Invariably, they will give rise to symmetric positive definite boundary element Galerkin matrices.

The notation $b_{*}$ in (3.8) is telling, because these will be the bilinear forms providing $\mathrm{b}$ in the operator preconditioning approach. Thus, most of the ingredients demanded by Theorem 2.1 are at our disposal now. We list them in Table 3.2.1 using the notation of Theorem 2.1 


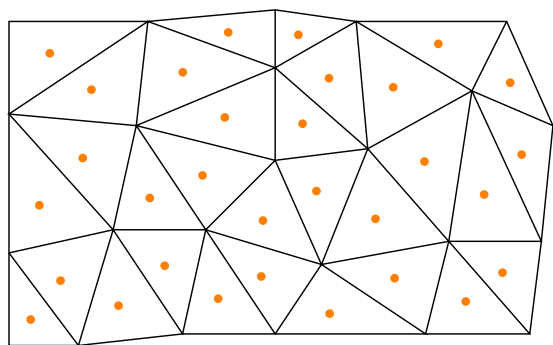

(a) Primal mesh $\Gamma_{h}$

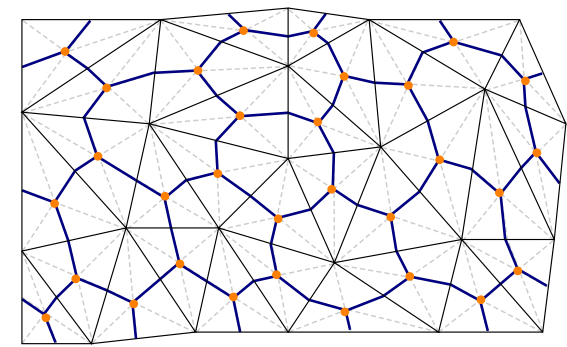

(b) Dual mesh $\hat{\Gamma}_{h}$

Fig. 4.1: Primal and dual meshes for (EDP). Black lines represent primal edges, dashed gray lines barycentric ones and blue lines are used to highlight dual edges. Orange dots mark the locations of degrees of freedom (DOFs) in $X_{h}=\mathcal{S}^{-1,0}\left(\Gamma_{h}\right)$ and $Y_{h}=\mathcal{S}^{0,1}\left(\widehat{\Gamma}_{h}\right)$.

4. Stable discrete $L^{2}$-duality. What is conspicuously missing in Table 3.2 .1 is the choice for $Y_{h}$. Recall that the assumptions of Theorem 2.1 imply $\operatorname{dim} X_{h}=\operatorname{dim} Y_{h}$, which rules out the use of $Y_{h}=\mathcal{S}_{0}^{0,1}\left(\Gamma_{h}\right)$ in the case (ENP). This problem is also encountered on closed surfaces and there it has been solved by means of boundary element spaces on dual meshes. In this section we elaborate this solution for screens.

4.1. Boundary element spaces on dual meshes. We build the dual meshes based on a barycentric refinement following Steinbach [23, Chap. 2.2] and Buffa and Christiansen [4. Starting from a triangular mesh $\Gamma_{h}$ of the screen as introduced in 1.3 dubbed primal mesh, we introduce its barycentric refinement $\bar{\Gamma}_{h}$, a finer triangular mesh obtained by splitting each triangle of $\Gamma_{h}$ into six smaller ones by adding the barycenter and all midpoints of edges as new vertices. Then, the cells of the dual mesh $\widehat{\Gamma}_{h}$ are (curved) polygons, each obtained by merging all those cells of $\bar{\Gamma}_{h}$ abutting a single node of the primal mesh. The nodes of $\widehat{\Gamma}_{h}$ coincide with the barycenters of cells of $\Gamma_{h}$. Specimens of primal and dual meshes are displayed in Figures 4.1 and 4.3 ,

Boundary element spaces on $\widehat{\Gamma}_{h}$, which will eventually serve as the spaces $Y_{h}$ for operator preconditioning, are spanned by linear combinations of basis functions of boundary element spaces $\bar{Y}_{h}$ built on $\bar{\Gamma}_{h}$. Now we outline this construction of $Y_{h}$ for our two main cases.

4.1.1. Case (EDP), (1.4). Recall from Subsection 1.3 that in this case we use $X_{h}=\mathcal{S}^{-1,0}\left(\Gamma_{h}\right) \subset \widetilde{H}^{-1 / 2}(\Gamma)$ and have to satisfy both $Y_{h} \subset H^{1 / 2}(\Gamma)$ and $\operatorname{dim} Y_{h}=$ $\operatorname{dim} X_{h}$, which is equal to the number of cells of $\Gamma_{h}$. We choose $Y_{h}$ as a subspace of the standard boundary element space on the barycentric refinement:

$$
\bar{Y}_{h}=\mathcal{S}^{0,1}\left(\bar{\Gamma}_{h}\right):=\text { "space of continuous piecewise linear functions on } \bar{\Gamma}_{h} \text { ". }
$$

The basis functions $\phi_{\tau}, \tau \in \Gamma_{h}$, of $Y_{h}$ are associated with the barycenters of primal cells and can be specified as local linear combinations of nodal basis functions of $\mathcal{S}^{0,1}\left(\bar{\Gamma}_{h}\right)$. In particular, the basis function $\phi_{\tau}, \tau \in \Gamma_{h}$, is a linear combination of those nodal basis functions $\bar{\phi}_{v}$ of $\mathcal{S}^{0,1}\left(\bar{\Gamma}_{h}\right)$ that belong to vertices $v$ of $\bar{\Gamma}_{h}$ that are contained in $\bar{\tau}$.

$$
\phi_{\tau}:=\sum_{v \in \bar{\tau}} c_{v} \bar{\phi}_{v}, \quad \tau \in \Gamma_{h} .
$$


The coefficient $c_{v}$ associated to a barycentric vertex $v$ is given by $c_{v}=\frac{1}{N_{v}}$, where $N_{v}$ equals the number of primal elements that are adjacent to $v$, which is also the number of dual nodal basis functions to which $\bar{\phi}_{v}$ will contribute. For the nodes $v$ at barycenters, we know that $c_{v}$ will always be equal to one. Analogously, the weight is always $\frac{1}{2}$ for the internal mid-edge nodes and 1 for those lying on $\partial \Gamma$. These weights are illustrated in Figure 4.2. They ensure that constant functions belong to

$$
Y_{h}=\mathcal{S}^{0,1}\left(\widehat{\Gamma}_{h}\right):=\operatorname{span}\left\{\phi_{\tau}: \tau \in \Gamma_{h}\right\} \subset H^{1 / 2}(\Gamma) .
$$
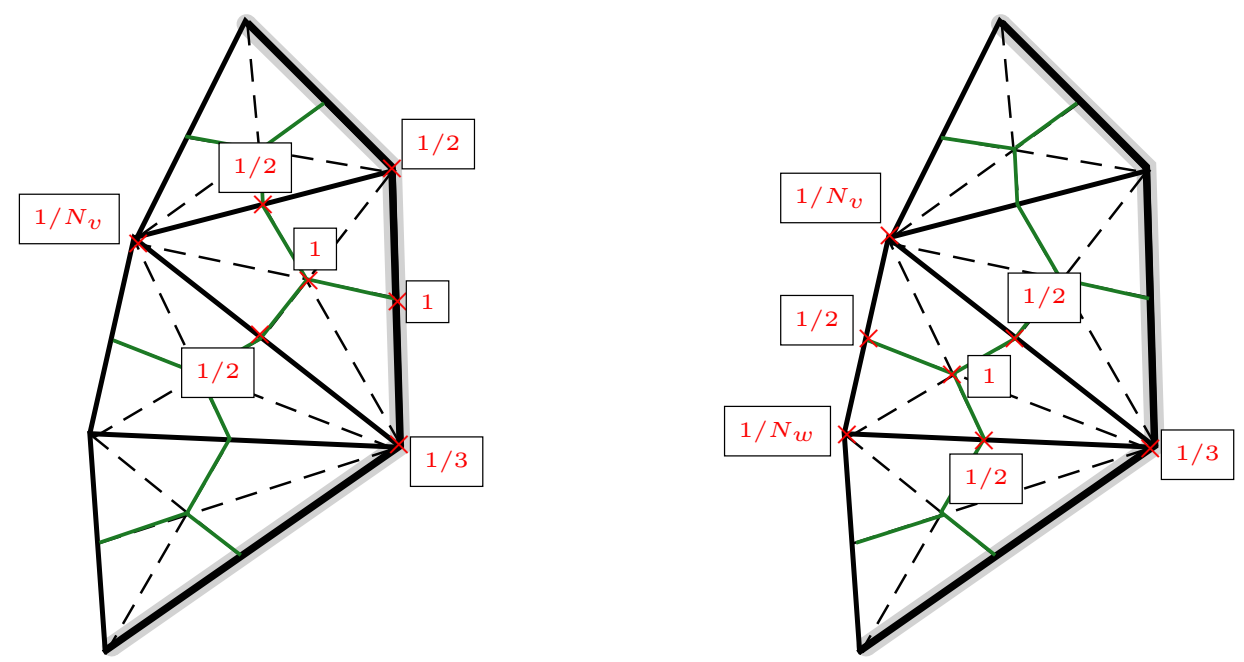

Fig. 4.2: Illustration of the linear combination coefficients to build $Y_{h}=\mathcal{S}^{0,1}\left(\widehat{\Gamma}_{h}\right)$, case (EDP), see also [4, Fig. 4]. On the left, we present the case when the primal cell has an edge on the boundary $\partial \Gamma$. On the right, we show the case when the primal cell has a single node located on $\partial \Gamma$. $N_{v}$ (resp. $N_{w}$ ) stands for the number of triangles sharing node $v$ (resp. $w$ ). In both situations, $\partial \Gamma$ is indicated by the thick gray line. We use black lines to draw primal edges, green lines for dual edges. Red crosses designate barycentric nodes.

4.1.2. Case (ENP), (1.6). In this case $X_{h}=\mathcal{S}_{0}^{0,1}\left(\Gamma_{h}\right) \subset \widetilde{H}^{1 / 2}(\Gamma)$ and $\operatorname{dim} X_{h}$ agrees with the number of interior vertices of $\Gamma_{h}$. We introduce $Y_{h}$ as subspace of

$$
\bar{Y}_{h}=\mathcal{S}^{-1,0}\left(\bar{\Gamma}_{h}\right):=\text { "space of piecewise constant functions on } \bar{\Gamma}_{h} . "
$$

and characterize it as the span of suitable basis functions. For each interior vertex $p$ of the primal mesh we introduce

$$
\phi_{p} \in Y_{h}, \quad \phi_{p}(\mathbf{x}):= \begin{cases}1, & \text { if } \mathbf{x} \text { belongs to the dual cell containing } p, \\ 0, & \text { elsewhere in } \Gamma,\end{cases}
$$

and set

$$
Y_{h}=\mathcal{S}_{*}^{-1,0}\left(\widehat{\Gamma}_{h}\right):=\operatorname{span}\left\{\phi_{p}: p \text { vertex of } \Gamma_{h}, p \notin \partial \Gamma\right\}
$$




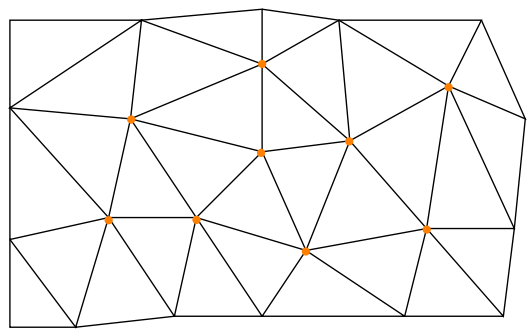

(a) Primal mesh $\Gamma_{h}$

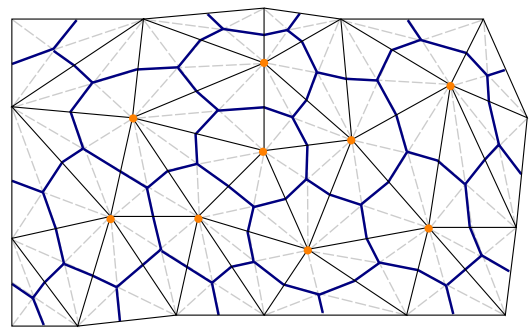

(b) Dual mesh $\hat{\Gamma}_{h}$

Fig. 4.3: Primal and dual meshes for case (ENP). Black lines represent primal edges, dashed gray lines barycentric ones, and blue lines are used to highlight dual edges. Orange dots mark the DOFs in $X_{h}=\mathcal{S}_{0}^{0,1}\left(\Gamma_{h}\right)$ and $Y_{h}=\mathcal{S}_{*}^{-1,0}\left(\widehat{\Gamma}_{h}\right)$.

Hence, $Y_{h}$ is spanned by the characteristic functions of only the interior cells of $\widehat{\Gamma}_{h}$, see Figure 4.3. Matching dimensions $\operatorname{dim} Y_{h}=\operatorname{dim} X_{h}$ is immediate.

REMARK 2. Let us review an implementation aspect of operator preconditioning in the dual mesh setting: Call $\mathbf{C}_{d}$ the sparse matrix representation of the inclusion operator $\bar{Y}_{h} \rightarrow Y_{h}$ given by the linear combinations discussed above. We construct the Galerkin matrix $\mathbf{B}_{h}$ of the bilinear form $\mathrm{b}$ over the dual mesh $\widehat{\Gamma}_{h}$ as follows: If $\mathbf{B}_{b}$ stands for the Galerkin matrix of $\mathrm{b}$ w.r.t. $\bar{Y}_{h}$ built on the barycentric refinement $\bar{\Gamma}_{h}$, then we can compute the Galerkin matrix for $\mathrm{b}$ on $Y_{h}$ as $\mathbf{B}_{h}=\mathbf{C}_{d} \mathbf{B}_{b} \mathbf{C}_{d}^{T}$.

In all cases we can regard $X_{h}$ as a subspace of a standard boundary element space $\bar{X}_{h}$ on the barycentric refinement $\bar{\Gamma}_{h}$. Introduce the sparse matrix representation $\mathbf{C}_{p}$ of the canonical embedding $\bar{X}_{h} \rightarrow X_{h}$ connecting local basis functions of $\bar{X}_{h}$ with those of $X_{h}$. Further, let $\mathbf{M}_{b}$ be the mass matrix, that is, the Galerkin matrix for $\mathrm{t}$ computed on the barycentric mesh. By using the above matrices, it is clear that the Galerkin matrix $\mathbf{T}_{h}$ associated to the $L^{2}$-duality pairing $\mathrm{t}$ is given by $\mathbf{T}_{h}=\mathbf{C}_{p} \mathbf{M}_{b} \mathbf{C}_{d}^{T}$ [15, Sect. 3.3-3.4].

\subsection{Stability of Discrete Duality Pairings on Non-Uniform Meshes.} As mentioned before, solutions of screen problems have a singular behavior near the boundary that can be resolved by refining the mesh towards it. This motivates our interest in applying the operator preconditioning strategy to non-uniform meshes. A key assumption of Theorem 2.1 is a discrete inf-sup condition for the $L^{2}(\Gamma)$-duality pairing $\mathrm{t}=\langle\cdot, \cdot\rangle_{\Gamma}$, and the spaces $X_{h}$ and $Y_{h}$ from Table 3.2.1, (4.2), (4.3).

In order to verify it, we extend the theory developed by O. Steinbach in 23 to spaces $\widetilde{H}^{ \pm 1 / 2}(\Gamma)$ and their corresponding dual spaces. Let us consider a given primal mesh $\Gamma_{h}$, and denote the size of an arbitrary cell $\tau \in \Gamma_{h}$ by $h_{\tau}$. Let $V_{h}$ be a boundary element space equipped with the standard nodal basis, for which we generically write $\left\{\varphi_{1}, \ldots, \varphi_{M}\right\}, M:=\operatorname{dim} V_{h}$. Concretely,

$$
\begin{array}{ll}
\text { for }(\mathbf{E D P}): & V_{h}:=\mathcal{S}^{0,1}\left(\Gamma_{h}\right) \subset H^{1 / 2}(\Gamma), \\
\text { for }(\mathbf{E N P}): & V_{h}:=\mathcal{S}_{0}^{0,1}\left(\Gamma_{h}\right) \subset \widetilde{H}^{1 / 2}(\Gamma) .
\end{array}
$$

Let $\omega_{k}:=\operatorname{supp}\left\{\varphi_{k}\right\}$. As a consequence of local quasi-uniformity, we can introduce for each nodal basis function $\varphi_{k} \in V_{h}$ an associated mesh size $\hat{h}_{k}$ satisfying

$$
\frac{1}{c_{Q}} \leq \frac{\hat{h}_{k}}{h_{\tau}} \leq c_{Q} \quad \text { for all } \tau \in \Gamma_{h} \text { such that } \tau \cap \omega_{k} \neq \emptyset, k=1, \ldots, M
$$


with a constant $c_{Q} \geq 1$ independent of $h \in \mathbb{H}$.

For an arbitrary (open) cell $\tau \in \Gamma_{h}$, define the index set of "adjacent basis functions"

$$
J(\tau):=\left\{k \in\{1, \ldots, M\}: \omega_{k} \cap \tau \neq \emptyset\right\} .
$$

Assumption 4.1 ([23, Eq. (2.30)]). We assume that the primal mesh $\Gamma_{h}$ satisfies the following local mesh condition:

$$
\frac{51}{7}-\sqrt{\sum_{k_{1} \in J(\tau)} \hat{h}_{k_{1}} \cdot \sum_{k_{2} \in J(\tau)} \hat{h}_{k_{2}}^{-1}} \geq c_{0}>0 \quad \forall \tau \in \Gamma_{h},
$$

with a global positive constant $c_{0}$.

REMARK 3. We point out that Assumption 4.1 turns out to be a mild condition and will always hold on meshes for which the local change in meshwidth is moderate. This can be verified as follows: From (4.4) we have that for each $k$ and $\tau \in \Gamma_{h}$ such that $\tau \cap \omega_{k} \neq \emptyset$ it holds that $\hat{h}_{k} \leq c_{Q} h_{\tau}$. Then

$$
\frac{51}{7}-\sqrt{\sum_{k_{1} \in J(\tau)} \hat{h}_{k_{1}} \sum_{k_{2} \in J(\tau)} \hat{h}_{k_{2}}^{-1}} \geq \frac{51}{7}-\sqrt{\left(\# J(\tau) c_{Q} h_{\tau}\right) \sum_{k_{2} \in J(\tau)} \hat{h}_{k_{2}}^{-1}} \forall \tau \in \Gamma_{h} .
$$

Use (4.4) again and conclude $\frac{h_{\tau}}{\hat{h}_{k}} \leq c_{Q}$ for any $k$ and $\tau$ such that $\tau \cap \omega_{k} \neq \emptyset$. We apply this and get

$$
\frac{51}{7}-\sqrt{\sum_{k_{1} \in J(\tau)} \hat{h}_{k_{1}} \sum_{k_{2} \in J(\tau)} \hat{h}_{k_{2}}^{-1}} \geq \frac{51}{7}-\sqrt{\left(\# J(\tau) c_{Q}\right)^{2}} \quad \forall \tau \in \Gamma_{h} .
$$

Since $\# J(\tau) \leq 3$ for all $\tau \in \Gamma_{h}$, Assumption 4.1 holds, if

$$
2.429 \approx \frac{51}{21} \geq c_{Q} \geq 1, \quad \forall \tau \in \Gamma_{h} .
$$

Moreover, in Section 6. Table 6.1.4, we list optimal lower bounds for $c_{0}$ for sequences of meshes used in our numerical experiments. These figures never get close to zero.

TheOrem 4.2. Let Assumptions 1.1 and 4.1 be satisfied. Then, for the following combinations of discrete spaces:

$$
\begin{array}{lll}
(\boldsymbol{E D P}): & X_{h}=\mathcal{S}^{-1,0}\left(\Gamma_{h}\right) \subset X=\widetilde{H}^{-1 / 2}(\Gamma), & Y_{h}=\mathcal{S}^{0,1}\left(\widehat{\Gamma}_{h}\right) \subset Y=H^{1 / 2}(\Gamma), \\
(\boldsymbol{E N P}): & X_{h}=\mathcal{S}_{0}^{0,1}\left(\Gamma_{h}\right) \subset X=\widetilde{H}^{1 / 2}(\Gamma), & Y_{h}=\mathcal{S}_{*}^{-1,0}\left(\widehat{\Gamma}_{h}\right) \subset Y=H^{-1 / 2}(\Gamma),
\end{array}
$$

the discrete inf-sup condition

$$
\sup _{v_{h} \in Y_{h}} \frac{\left|\left\langle w_{h}, v_{h}\right\rangle_{\Gamma}\right|}{\left\|v_{h}\right\|_{Y}} \geq \frac{1}{c_{s}}\left\|w_{h}\right\|_{X}, \quad \forall w_{h} \in X_{h},
$$

holds with a positive constant $c_{s}$ independent of $h$.

Proof. We restrict ourselves to the case (ENP). Case EDP follows analogously as can be found in the preprint [12, Sect. 3.3]. We adapt and extend the proof of [23, Theorem 2.1 and 2.2]. The required extensions are analogous to those developed in 11] for the case of the segment and are discussed here for the sake of completeness. 
(1 From [23, Lemma 1.7] we learn that Assumption 4.1 guarantees

$$
\sup _{\psi_{h} \in \mathcal{S}_{*}^{-1,0}\left(\hat{\Gamma}_{h}\right)} \frac{\left|\left\langle\psi_{h}, w_{h}\right\rangle_{\Gamma}\right|}{\left\|\psi_{h}\right\|_{L^{2}(\Gamma)}} \geq c_{s t}\left\|w_{h}\right\|_{L^{2}(\Gamma)}, \quad \forall w_{h} \in \mathcal{S}_{0}^{0,1}\left(\Gamma_{h}\right)
$$

with $c_{s t}>0$ independent of $h$. Thus, the generalized Galerkin $L^{2}$-Projection $\tilde{Q}_{h}$ : $L^{2}(\Gamma) \rightarrow \mathcal{S}_{0}^{0,1}\left(\Gamma_{h}\right)$, defined for $u \in L^{2}(\Gamma)$ as follows:

$$
\left\langle\tilde{Q}_{h} u, \phi_{h}\right\rangle_{\Gamma}=\left\langle u, \phi_{h}\right\rangle_{\Gamma}, \quad \forall \phi_{h} \in \mathcal{S}_{*}^{-1,0}\left(\hat{\Gamma}_{h}\right),
$$

is well-defined and bounded in $L^{2}(\Gamma)$.

(2) To show the $H^{1}(\Gamma)$-stability of $\tilde{Q}_{h}$, we employ a quasi-interpolation operator, as in [23, Section 1.5]. Let us define the local trial space related to the local basis function $\varphi_{k}$ as

$$
X_{h}\left(\omega_{k}\right):=\operatorname{span}\left\{\left.\varphi_{j}\right|_{\omega_{k}}: \varphi_{j} \in \mathcal{S}_{0}^{0,1}\left(\Gamma_{h}\right)\right\}
$$

Let $Q_{h}^{k}$ denote the standard $L^{2}(\Gamma)$-orthogonal projection onto $X_{h}\left(\omega_{k}\right)$, such that for $u \in L^{2}\left(\omega_{k}\right)$

$$
\left\langle Q_{h}^{k} u, v_{h}\right\rangle_{\omega_{k}}=\left\langle u, v_{h}\right\rangle_{\omega_{k}}, \quad \forall v_{h} \in X_{h}\left(\omega_{k}\right) .
$$

Due to Assumption 1.1. $Q_{h}^{k}$ satisfies the following stability and quasi-optimal error estimates [23, Section 1.5]: there is a constant $c_{s t}^{\text {loc }}>0$ independent of $h$

$$
\begin{aligned}
\left\|Q_{h}^{k} u\right\|_{L^{2}\left(\omega_{k}\right)} & \leq\|u\|_{L^{2}\left(\omega_{k}\right)}, & & \forall u \in L^{2}\left(\omega_{k}\right), \\
\|\left(\text { Id }-Q_{h}^{k}\right) u \|_{L^{2}\left(\omega_{k}\right)} & \leq c_{s t}^{l o c} \hat{h}_{k}|u|_{H^{1}\left(\omega_{k}\right)}, & & \forall u \in H^{1}\left(\omega_{k}\right) .
\end{aligned}
$$

Moreover, local quasi-uniformity gives us the following stability estimate via inverse estimates

$$
\exists \tilde{c}_{s t}^{l o c}>0:\left\|Q_{h}^{k} u\right\|_{H^{1}\left(\omega_{k}\right)} \leq \tilde{c}_{s t}^{l o c} \hat{h}_{k}\|u\|_{H^{1}\left(\omega_{k}\right)} \text {, for all } u \in H^{1}\left(\omega_{k}\right) .
$$

Then, it is possible to define a quasi-interpolation operator $P_{h}: L^{2}(\Gamma) \rightarrow \mathcal{S}_{0}^{0,1}\left(\Gamma_{h}\right)$ by

$$
\left(P_{h} u\right)(x):=\sum_{k=1}^{M}\left(Q_{h}^{k} u\right)\left(x_{k}\right) \cdot \varphi_{k}(x),
$$

which is also a projection onto $X_{h}$. Furthermore, $P_{h}$ enjoys the following important properties: extending Lemma 1.9 of [23] to $H_{0}^{1}(\Gamma)$ confirms that there exists a constant $c_{p 1}>0$ independent of $h$ such that, for all $u \in H_{0}^{1}(\Gamma)$, it holds

$$
\begin{aligned}
\left\|\left(\mathbf{I d}-P_{h}\right) u\right\|_{L^{2}(\tau)} & \leq c_{p 1} \sum_{k \in J(\tau)} \hat{h}_{k}|u|_{H^{1}\left(\omega_{k}\right)}, \quad \forall \tau \in \Gamma_{h}, \\
\left\|P_{h} u\right\|_{H^{1}(\Gamma)} & \leq c_{p 1}\|u\|_{H^{1}(\Gamma)}, \\
\sum_{k=1}^{M} \hat{h}_{k}^{-2}\left\|\left(\mathbf{l d}-P_{h}\right) u\right\|_{L^{2}\left(\omega_{k}\right)}^{2} & \leq c_{p 1}\|u\|_{H^{1}(\Gamma)}^{2} .
\end{aligned}
$$


(3) Extend Lemma 2.3 of [23] to the spaces $X_{h}=\mathcal{S}_{0}^{0,1}\left(\Gamma_{h}\right), Y_{h}=\mathcal{S}_{*}^{-1,0}\left(\hat{\Gamma}_{h}\right)$ by local computations (see Appendix of preprint [15]). Then, under Assumption 4.1 and by writing $\phi_{k}, k=1, \ldots, M$, for the basis functions of $\mathcal{S}_{*}^{-1,0}\left(\hat{\Gamma}_{h}\right)(c f$. (4.3)), we obtain

$$
\sum_{\tau \in \Gamma_{h}} h_{\tau}^{-2}\left\|v_{h}\right\|_{L^{2}(\tau)}^{2} \leq c_{p 2} \sum_{k=1}^{M}\left[\frac{\left\langle v_{h}, \phi_{k}\right\rangle_{\Gamma}}{\hat{h}_{k}\left\|\phi_{k}\right\|_{L^{2}(\Gamma)}}\right]^{2}, \quad \forall v_{h} \in \mathcal{S}_{0}^{0,1}\left(\Gamma_{h}\right),
$$

with a constant $c_{p 2}>0$ independent of the meshwidth.

$(4$ Combining all these results, we find that under Assumptions 1.1 and 4.1 the generalized $L^{2}(\Gamma)$-orthogonal projection $\tilde{Q}_{h}: H_{0}^{1}(\Gamma) \rightarrow X_{h}=\mathcal{S}_{0}^{0,1}\left(\Gamma_{h}\right)$ as defined in (4.9) satisfies

$$
\left\|\tilde{Q}_{h} u\right\|_{H^{1}(\Gamma)} \leq \tilde{c}_{s t}\|u\|_{H^{1}(\Gamma)}, \quad \forall u \in H_{0}^{1}(\Gamma),
$$

with $\tilde{c}_{s t}>0$ being a constant independent of the meshwidth. This emerges from the following chain of estimates:

$$
\begin{aligned}
\left\|\tilde{Q}_{h} u\right\|_{H^{1}(\Gamma)}^{2} & \leq 2\left\{\left\|P_{h} u\right\|_{H^{1}(\Gamma)}^{2}+\left\|\left(\tilde{Q}_{h}-P_{h}\right) u\right\|_{H^{1}(\Gamma)}^{2}\right\} \\
& \leq 2\left\{c_{p 1}\|u\|_{H^{1}(\Gamma)}^{2}+\left\|\left(\tilde{Q}_{h}-P_{h}\right) u\right\|_{H^{1}(\Gamma)}^{2}\right\} \\
(\text { Inverse estimate) } & \leq 2\left\{c_{p 1}\|u\|_{H^{1}(\Gamma)}^{2}+\sum_{\tau \in \Gamma_{h}} h_{\tau}^{-2}\left\|\left(\tilde{Q}_{h}-P_{h}\right) u\right\|_{L^{2}(\tau)}^{2}\right\} \\
& \leq 2\left\{c_{p 1}\|u\|_{H^{1}(\Gamma)}^{2}+c_{p 2} \sum_{k=1}^{M}\left[\frac{\left\langle\left(\tilde{Q}_{h}-P_{h}\right) u, \phi_{k}\right\rangle}{\hat{h}_{k}\left\|\phi_{k}\right\|_{L^{2}(\Gamma)}}\right]^{2}\right\} \\
& \leq 2\left\{c_{p 1}\|u\|_{H^{1}(\Gamma)}^{2}+c_{p 2} \sum_{k=1}^{M}\left[\frac{\left\langle\left(\mathrm{Id}-P_{h}\right) u, \phi_{k}\right\rangle_{\omega_{k}}}{\hat{h}_{k}\left\|\phi_{k}\right\|_{L^{2}(\Gamma)}}\right]\right\} \\
& \leq 2\left\{c_{p 1}\|u\|_{H^{1}(\Gamma)}^{2}+c_{p 2} \sum_{k=1}^{M} \hat{h}_{k}^{-2}\left\|\left(\mathrm{Id}-P_{h}\right) u\right\|_{L^{2}\left(\omega_{k}\right)}^{2}\right\} \\
& \leq \tilde{c}_{s t}\|u\|_{H^{1}(\Gamma)}^{2} .
\end{aligned}
$$

(5 It is well known that $\widetilde{H}^{1 / 2}(\Gamma)$ can be defined by interpolating between $L^{2}(\Gamma)$ and $H_{0}^{1}(\Gamma)$; see [17, Thm. 11.7]. Thus, by interpolation of bounded linear operators we obtain from $L^{2}$-stability and (4.19) that

$$
\left\|\tilde{Q}_{h} u\right\|_{\widetilde{H}^{1 / 2}(\Gamma)} \leq c_{B}\|u\|_{\widetilde{H}^{1 / 2}(\Gamma)}, \quad \forall u \in \widetilde{H}^{1 / 2}(\Gamma) .
$$

(6 Introduce the projection operators $\Pi_{h}: \widetilde{H}^{1 / 2}(\Gamma) \rightarrow \mathcal{S}_{*}^{-1,0}\left(\hat{\Gamma}_{h}\right) \subset H^{-1 / 2}(\Gamma)$, satisfying

$$
\left\langle\Pi_{h} u, w_{h}\right\rangle_{\Gamma}=\left(u, w_{h}\right)_{\widetilde{H}^{1 / 2}(\Gamma)}, \quad \forall w_{h} \in \mathcal{S}_{0}^{0,1}\left(\Gamma_{h}\right),
$$

where $(\cdot, \cdot)_{\widetilde{H}^{1 / 2}(\Gamma)}$ denotes the $\widetilde{H}^{1 / 2}(\Gamma)$-inner product. Notice that (4.8) guarantees that $\Pi_{h}$ is well-defined. 


\begin{tabular}{|c|c|c|c|c|c|c|}
\hline BVP & $\mathrm{a}$ & $\mathrm{b}$ & $X$ & $Y$ & $X_{h}$ & $Y_{h}$ \\
\hline \hline$($ EDP $)$ & $\mathrm{av}_{, \mathbb{D}_{a}}$ & $\mathrm{~b}_{\overline{\mathrm{W}}, a}$ & $\widetilde{H}^{-1 / 2}(\Gamma)$ & $H^{1 / 2}(\Gamma)$ & $\mathcal{S}^{-1,0}\left(\Gamma_{h}\right)$ & $\mathcal{S}^{0,1}\left(\widehat{\Gamma}_{h}\right)$ \\
\hline (ENP) & $\mathrm{a}_{\mathrm{W}, \mathbb{D}_{a}}$ & $\mathrm{~b}_{\overline{\mathrm{V}}, a}$ & $\widetilde{H}^{1 / 2}(\Gamma)$ & $H^{-1 / 2}(\Gamma)$ & $\mathcal{S}_{0}^{0,1}\left(\Gamma_{h}\right)$ & $\mathcal{S}_{*}^{-1,0}\left(\widehat{\Gamma}_{h}\right)$ \\
\hline
\end{tabular}

Table 4.2.1: Completion of Table 4.2.1. Components of the $L^{2}(\Gamma)$-based dual mesh operator preconditioning approach for variational boundary integral equations on $\Gamma=\mathbb{D}_{a}$.

By the definition of the dual norm and continuity of $\tilde{Q}_{h}$, one can derive from (4.21) that

$$
\begin{aligned}
& \left\|\Pi_{h} u\right\|_{H^{-1 / 2}(\Gamma)}=\sup _{0 \neq w \in \widetilde{H}^{1 / 2}(\Gamma)} \frac{\left|\left\langle\Pi_{h} u, w\right\rangle_{\Gamma}\right|}{\|w\|_{\widetilde{H}^{1 / 2}(\Gamma)}}=\sup _{0 \neq w \in \widetilde{H}^{1 / 2}(\Gamma)} \frac{\left|\left\langle\Pi_{h} u, \tilde{Q}_{h} w\right\rangle_{\Gamma}\right|}{\|w\|_{\widetilde{H}^{1 / 2}(\Gamma)}} \\
& \quad \leq c_{B} \sup _{0 \neq w \in \widetilde{H}^{1 / 2}(\Gamma)} \frac{\left|\left\langle\Pi_{h} u, \tilde{Q}_{h} w\right\rangle_{\Gamma}\right|}{\left\|\tilde{Q}_{h} w\right\|_{\widetilde{H}^{1 / 2}(\Gamma)}}=c_{B} \sup _{0 \neq w \in \widetilde{H}^{1 / 2}(\Gamma)} \frac{\left|\left(u, \tilde{Q}_{h} w\right)_{\widetilde{H}^{1 / 2}(\Gamma)}\right|}{\left\|\tilde{Q}_{h} w\right\|_{\widetilde{H}^{1 / 2}(\Gamma)}} \\
& \leq c_{B}\|u\|_{\widetilde{H}^{1 / 2}(\Gamma)},
\end{aligned}
$$

for all $u \in \widetilde{H}^{1 / 2}(\Gamma)$.

(7) Finally, by the above inequality, for any $w_{h} \in \mathcal{S}_{0}^{0,1}\left(\Gamma_{h}\right)$ we obtain the assertion

$$
\begin{aligned}
\left\|w_{h}\right\|_{\widetilde{H}^{1 / 2}(\Gamma)} & =\frac{\left|\left(w_{h}, w_{h}\right)_{\widetilde{H}^{1 / 2}(\Gamma)}\right|}{\left\|w_{h}\right\|_{\widetilde{H}^{1 / 2}(\Gamma)}}=\frac{\left|\left\langle w_{h}, \Pi_{h} w_{h}\right\rangle_{\Gamma}\right|}{\left\|w_{h}\right\|_{\widetilde{H}^{1 / 2}(\Gamma)}} \\
& \leq c_{B} \frac{\left|\left\langle w_{h}, \Pi_{h} w_{h}\right\rangle_{\Gamma}\right|}{\left\|\Pi_{h} w_{h}\right\|_{H^{-1 / 2}(\Gamma)}} \leq c_{B} \sup _{0 \neq v_{h} \in \mathcal{S}_{*}^{-1,0}\left(\hat{\Gamma}_{h}\right)} \frac{\left|\left\langle w_{h}, v_{h}\right\rangle_{\Gamma}\right|}{\left\|v_{h}\right\|_{H^{-1 / 2}(\Gamma)}} .
\end{aligned}
$$

(

The estimate of Theorem 4.2 ultimately justifies the choice of the spaces $Y_{h}$ in Section 4.1 and confirms the crucial stability requirement for the operator preconditioning approach summarized now in Table 4.2.1

COROLLARY 4.3. Borrowing notations from Theorem [2.1, consider the Galerkin boundary element discretization and the $L^{2}(\Gamma)$-based operator preconditioning approach for (1.4) (EDP) and (1.6) (ENP) as detailed in Table 4.2.1. Under Assumptions 1.1 and 4.1, the spectral condition number of the preconditioned Galerkin matrix $\mathbf{T}_{h}^{-1} \mathbf{B}_{h} \mathbf{T}_{h}^{-H} \mathbf{A}_{h}$ is bounded independently of $h \in \mathbb{H}$.

\section{Operator Preconditioning on General Screens.}

5.1. Mapped Screens. We now extend the operator preconditioning strategy to more general screens $\Gamma \subset \mathbb{R}^{3}$, for which there is a bi-Lipschitz continuous 22. Def. 2.2.5] piecewise $C^{1}$-mapping $\widetilde{\phi}: \overline{\mathbb{D}}_{1} \times[-1,1] \rightarrow \mathbb{R}^{3}$ such that $\phi\left(\overline{\mathbb{D}}_{1}\right)=\bar{\Gamma}$ for $\phi:=\widetilde{\phi}_{\mid \mathbb{\mathbb { D }}_{1} \times\{0\}}$. This ensured that $\Gamma$ is an orientable two-dimensional $C^{1}$-piecewise manifold with boundary $\partial \Gamma=\phi\left(\partial \mathbb{D}_{1}\right)$. 
- Example 1: In 3D cylindrical coordinates $(r, \theta, z)$ define

$$
\widetilde{\phi}\left[\begin{array}{l}
r \\
\theta \\
z
\end{array}\right]=\left[\begin{array}{c}
r / \max (|\cos \varphi|,|\sin \varphi|) \\
\theta \\
z
\end{array}\right],
$$

for $r \geq 0,0 \leq \theta \leq 2 \pi$, and $z \in \mathbb{R}$. Then $\widetilde{\phi}$ satisfies the above assumptions and maps the unit disk to a square $\phi\left(\mathbb{D}_{1}\right)=[-1,1]^{2}$.

- Example 2: If $f \in C^{1}\left(\overline{\mathbb{D}}_{1}, \mathbb{R}\right)$ and

$$
\widetilde{\boldsymbol{\phi}}\left[\begin{array}{l}
x_{1} \\
x_{2} \\
x_{3}
\end{array}\right]=\left[\begin{array}{c}
x_{1} \\
x_{2} \\
x_{3}+f\left(x_{1}, x_{2}\right)
\end{array}\right], \quad x_{1}, x_{2}, x_{3} \in \mathbb{R},
$$

then $\Gamma:=\phi\left(\mathbb{D}_{1}\right)$ yields the graph of $f$ over $\mathbb{D}_{1}$.

We consider the variational problems (1.4) and (1.6) on $\Gamma$. Writing $\phi^{*} v: \mathbb{D}_{1} \rightarrow \mathbb{R}$ for the pullback of a function $v: \Gamma \rightarrow \mathbb{R}$ to $\mathbb{D}_{1}$ under $\phi$ :

$$
\hat{v}(\hat{\mathbf{x}}):=\left(\phi^{*} v\right)(\hat{\mathbf{x}})=v(\phi(\hat{\mathbf{x}})), \quad \hat{\mathbf{x}} \in \mathbb{D}_{1}
$$

We recall from [18, Thm 3.23] that

$$
\phi^{*}: L^{2}(\Gamma) \rightarrow L^{2}\left(\mathbb{D}_{1}\right), \quad \phi^{*}: H^{1}(\Gamma) \rightarrow H^{1}\left(\mathbb{D}_{1}\right), \quad \phi^{*}: H_{0}^{1}(\Gamma) \rightarrow H_{0}^{1}\left(\mathbb{D}_{1}\right),
$$

are isomorphisms, since $\phi$ is bi-Lipschitz. By interpolation arguments in Sobolev scales, we immediately infer that

$$
\begin{aligned}
& \phi^{*}: H^{1 / 2}(\Gamma) \rightarrow H^{1 / 2}\left(\mathbb{D}_{1}\right), \\
& \phi^{*}: \widetilde{H}^{1 / 2}(\Gamma) \rightarrow \widetilde{H}^{1 / 2}\left(\mathbb{D}_{1}\right),
\end{aligned}
$$

are isomorphisms too. By duality, this will also hold for

$$
\begin{aligned}
& \phi^{*}: \widetilde{H}^{-1 / 2}(\Gamma) \rightarrow \widetilde{H}^{-1 / 2}\left(\mathbb{D}_{1}\right), \\
& \phi^{*}: H^{-1 / 2}(\Gamma) \rightarrow H^{-1 / 2}\left(\mathbb{D}_{1}\right) .
\end{aligned}
$$

5.2. Unit-Disk-Based Preconditioner for Mapped Screens. The idea is to rely on isomorphisms (5.3)-(5.6) to obtain suitable bilinear forms $b(\cdot, \cdot)$ for operator preconditioning according to Theorem 2.1. Also note that all constructions and results of Section 4 had already been formulated and proved for general screens as introduced above.

For the sake of Galerkin boundary element discretization, we equip $\Gamma$ with a uniformly shape regular family $\left\{\Gamma_{h}\right\}_{h \in \mathbb{H}}$ of surface meshes, obtained as the image of a family of meshes $\left\{\mathbb{D}_{h}\right\}_{h \in \mathbb{H}}$ of the unit disk $\mathbb{D}_{1}$ under $\phi$, i.e. $\Gamma_{h}=: \phi\left(\mathbb{D}_{h}\right)$, which is supposed to comply with Assumption 1.1 .

Case (EDP). We write $\bigvee_{\Gamma}$ to denote the weakly singular operator (1.2) on $\Gamma=$ $\phi\left(\mathbb{D}_{1}\right)$. Using the parametrization $\phi$, it can be pulled back to $\mathbb{D}_{1}: \bigvee_{\Gamma}^{*}=\left(\phi^{*}\right)^{\prime} \circ \mathrm{V}_{\Gamma} \circ \phi^{*}$, where $\left(\phi^{*}\right)^{\prime}$ denotes the adjoint operator of $\phi^{*}$. Hence, $\bigvee_{\Gamma}^{*}$ induces the bilinear form for $\hat{\sigma}, \hat{\mu} \in \widetilde{H}^{-1 / 2}\left(\mathbb{D}_{1}\right)$

$$
\mathrm{a}_{\mathrm{V}, \Gamma}^{*}(\hat{\sigma}, \hat{\mu}):=\frac{1}{4 \pi} \int_{\mathbb{D}_{1}} \int_{\mathbb{D}_{1}} \frac{\hat{\sigma}(\hat{\mathbf{y}}) \hat{\mu}(\hat{\mathbf{x}})}{\|\phi(\hat{\mathbf{x}})-\phi(\hat{\mathbf{y}})\|} g(\hat{\mathbf{x}}) g(\hat{\mathbf{y}}) d \mathbb{D}_{1}(\hat{\mathbf{x}}) d \mathbb{D}_{1}(\hat{\mathbf{y}}),
$$


Table 5.2.1: Components of $L^{2}(\Gamma)$-based dual mesh operator preconditioning for variational BIEs on $\Gamma=\phi\left(\mathbb{D}_{1}\right)$ under Assumptions 1.1 and 4.1 Recall that $\mathbb{D}_{h}$ denotes the primal mesh of $\mathbb{D}_{1}$ and here we write $\widehat{\mathbb{D}}_{h}$ for its dual mesh.

\begin{tabular}{|c|c|c|c|c|c|c|}
\hline BVP & $\mathrm{a}$ & $\mathrm{b}$ & $X$ & $Y$ & $X_{h}$ & $Y_{h}$ \\
\hline \hline$($ EDP $)$ & $\mathrm{a}_{\mathrm{V}, \Gamma}^{*}$ & $\mathrm{~b}_{\overline{\mathrm{W}}, 1}$ & $\widetilde{H}^{-1 / 2}\left(\mathbb{D}_{1}\right)$ & $H^{1 / 2}\left(\mathbb{D}_{1}\right)$ & $\mathcal{S}^{-1,0}\left(\mathbb{D}_{h}\right)$ & $\mathcal{S}^{0,1}\left(\widehat{\mathbb{D}}_{h}\right)$ \\
\hline$(\mathbf{E N P})$ & $\mathrm{a}_{\mathrm{W}, \Gamma}^{*}$ & $\mathrm{~b}_{\overline{\mathrm{V}}, 1}$ & $\widetilde{H}^{1 / 2}\left(\mathbb{D}_{1}\right)$ & $H^{-1 / 2}\left(\mathbb{D}_{1}\right)$ & $\mathcal{S}_{0}^{0,1}\left(\mathbb{D}_{h}\right)$ & $\mathcal{S}_{*}^{-1,0}\left(\widehat{\mathbb{D}}_{h}\right)$ \\
\hline
\end{tabular}

with $g(\hat{\mathbf{x}}):=\sqrt{\operatorname{det}\left(D \phi(\hat{\mathbf{y}})^{T} D \phi(\hat{\mathbf{y}})\right)}$, and $D \phi(\hat{\mathbf{x}})$ the Jacobian of $\phi$ in $\hat{\mathbf{x}} \in \mathbb{D}_{1}$. Thanks to (5.5), $a_{\mathrm{V}, \Gamma}^{*}$ is $\widetilde{H}^{-1 / 2}\left(\mathbb{D}_{1}\right)$-elliptic and continuous. Thus, we can employ $X_{h}=\mathcal{S}^{-1,0}\left(\mathbb{D}_{h}\right)$ for Galerkin discretization and $\mathrm{b}_{\overline{\mathrm{w}}, 1}$ from $(\underline{3.8 \mathrm{~b}})$ for operator preconditioning.

Case (ENP). Let us write $\mathrm{W}_{\Gamma}$ for the hypersingular operator (1.5) on the screen $\Gamma=\phi\left(\mathbb{D}_{1}\right)$ :

$$
\left(\mathrm{W}_{\Gamma} u\right)(\mathbf{x}):=\int_{\Gamma} k_{\mathrm{W}}(\mathbf{x}, \mathbf{y}) u(\mathbf{y}) d \Gamma(\mathbf{y}), \quad \mathbf{x} \in \Gamma, u \in \widetilde{H}^{1 / 2}(\Gamma)
$$

where $k_{\mathrm{W}}$ is the associated kernel. As for (EDP), we can use the parameterization of $\Gamma$ over $\mathbb{D}_{1}$ to define $\mathrm{W}_{\Gamma}^{*}:=\left(\phi^{*}\right)^{\prime} \circ \mathrm{W}_{\Gamma} \circ \phi^{*}$, which induces the bilinear form

$$
\mathrm{a}_{\mathrm{W}, \Gamma}^{*}(\hat{u}, \hat{v}):=\frac{1}{4 \pi} \int_{\mathbb{D}_{1}} \int_{\mathbb{D}_{1}} \frac{\operatorname{curl}_{\Gamma, \phi(\hat{\mathbf{x}})} \hat{u}(\hat{\mathbf{x}}) \cdot \operatorname{curl}_{\Gamma, \phi(\hat{\mathbf{y}})} \hat{v}(\hat{\mathbf{y}})}{\|\phi(\hat{\mathbf{x}})-\phi(\hat{\mathbf{y}})\|} g(\hat{\mathbf{x}}) g(\hat{\mathbf{y}}) d \mathbb{D}_{1}(\hat{\mathbf{x}}) d \mathbb{D}_{1}(\hat{\mathbf{y}})
$$

for $\hat{u}, \hat{v} \in \widetilde{H}^{1 / 2}\left(\mathbb{D}_{1}\right)$, and $g$ and $D \phi$ as before.

The norm equivalence implied by (5.4) gives $\widetilde{H}^{1 / 2}\left(\mathbb{D}_{1}\right)$-ellipticity and continuity of $\mathrm{a}_{\mathrm{W}, \Gamma}^{*}$. Therefore we can use $X_{h}=\mathcal{S}_{0}^{0,1}\left(\mathbb{D}_{h}\right)$ for Galerkin discretization and $\mathrm{b}_{\overline{\mathrm{v}}, 1}$ from (3.8a) for operator preconditioning.

COROLlaRY 5.1. Borrowing notations from Theorem [2.1, we consider the variational BIEs (EDP) and (ENP), and their Galerkin boundary element discretization according to Table [5.2.1. Then, the operator preconditioning strategy proposed in Table 5.2.1 yields h-uniformly bounded spectral condition number $\kappa\left(\mathbf{T}_{h}^{-1} \mathbf{B}_{h} \mathbf{T}_{h}^{-H} \mathbf{A}_{h}\right)$.

REMARK 4. The isomorphisms (5.3)-(5.6) imply equivalence of corresponding norms of a function $v$ on $\Gamma$ and its pullback $\phi^{*} v$. The constants in these equivalences will have a direct impact on the final bounds for the spectral condition numbers of the preconditioned Galerkin matrices through $\|\mathrm{a}\|$ and $c_{A}$ (see Theorem 2.1 for notations).

This means that Corollary 5.1 guarantees that the condition number (2.2) will remain h-uniformly bounded for the preconditioned Galerkin matrices of $\mathrm{V}_{\Gamma}$ and $\mathrm{W}_{\Gamma}$, albeit affected by a constant depending on the distortion effected by $\phi$. Crudely speaking, in case $\phi$ causes large distortions, that is, if $\|D \phi\|_{\infty}$ or $\left\|D \phi^{-1}\right\|_{\infty}$ are large, we should expect poorer performance of the preconditioner. This will be reflected in the numerical experiments in Section 6.2 through a pre-asymptotic phase in which the behavior of the preconditioner is not as good as expected. 
5.3. Shape-intrinsic preconditioners. Of course, it would be desirable to dispense with any mapping from $\mathbb{D}_{1}$ and to compute suitable $\mathrm{b}_{\overline{\mathrm{V}}, a}$ and $\mathrm{b}_{\overline{\mathrm{v}}, a}$ directly on the general screen $\Gamma$. We will briefly present considerations that are entirely heuristic so far, because a rigorous proof of their efficacy is not (yet) available. The idea is to take the cue from (3.1) and (3.2) and replace $S_{a}(\mathbf{x}, \mathbf{y})$ in these formulas with $S_{\Gamma}(\mathbf{x}, \mathbf{y})$, $\mathbf{x}, \mathbf{y} \in \Gamma$, such that $S_{\Gamma} \in L^{\infty}(\Gamma \times \Gamma), S_{\Gamma}(\mathbf{x}, \mathbf{y})=S_{\Gamma}(\mathbf{y}, \mathbf{x})$, and $S_{\Gamma}(\mathbf{x}, \mathbf{y}) \sim \sqrt{\operatorname{dist}(\mathbf{x}, \partial \Gamma)}$ for $\mathbf{x} \rightarrow \partial \Gamma, \mathbf{y} \notin \Gamma$. The resulting preconditioners will employ the following bilinear forms $\mathrm{b}_{\overline{\mathrm{V}}, a}$ and $\mathrm{b}_{\overline{\mathrm{W}}, a}$ on $\Gamma$ :

$$
\begin{aligned}
\mathrm{b}_{\overline{\mathrm{v}}, \Gamma}(\nu, \mu):= & \frac{2}{\pi^{2}} \int_{\Gamma} \int_{\Gamma} \frac{S_{\Gamma}(\mathbf{x}, \mathbf{y})}{\|\mathbf{x}-\mathbf{y}\|} \nu(\mathbf{x}) \mu(\mathbf{y}) d \Gamma(\mathbf{x}) d \Gamma(\mathbf{y}) \\
\mathrm{b}_{\overline{\mathrm{W}}, \Gamma}(u, v):= & \frac{2}{\pi^{2}} \int_{\Gamma} \int_{\Gamma} \frac{S_{\Gamma}(\mathbf{x}, \mathbf{y})}{\|\mathbf{x}-\mathbf{y}\|^{3}} \operatorname{curl}_{\Gamma, \mathbf{x}} u(\mathbf{x}) \cdot \operatorname{curl}_{\Gamma, \mathbf{y}} v(\mathbf{y}) d \Gamma(\mathbf{x}) d \Gamma(\mathbf{y}) \\
& +\alpha \overline{\mathrm{W}}\langle u, 1\rangle_{\Gamma}\langle v, 1\rangle_{\Gamma},
\end{aligned}
$$

for $\nu, \mu \in H^{-1 / 2}(\Gamma), u, v \in H^{1 / 2}(\Gamma)$, and with $\alpha_{\bar{W}} \in \mathbb{R}_{+}$bounded. In addition, we replaced the function $\omega^{-1}$ by the constant 1 in the correction term to simplify implementation. Finally, we combine the building blocks introduced in Sections 2 and प with the bilinear forms $\mathrm{b}_{\overline{\mathrm{v}}, \Gamma}$ and $\mathrm{b}_{\overline{\mathrm{W}}, \Gamma}$ to construct shape-intrinsic preconditioners, see Table 5.3.1. We refer to [28, Chapter 4] for a more extensive discussion.

Table 5.3.1: Components of Shape-aware preconditioning for variational BIEs on $\Gamma$.

\begin{tabular}{|c|c|c|c|c|c|c|}
\hline BVP & $\mathrm{a}$ & $\mathrm{b}$ & $X$ & $Y$ & $X_{h}$ & $Y_{h}$ \\
\hline \hline (EDP) & $\mathrm{a}_{\mathrm{V}, \Gamma}^{\mathrm{k}}$ & $\mathrm{b}_{\overline{\mathrm{W}}, \Gamma}$ & $\widetilde{H}^{-1 / 2}(\Gamma)$ & $H^{1 / 2}(\Gamma)$ & $\mathcal{S}^{-1,0}\left(\Gamma_{h}\right)$ & $\mathcal{S}^{0,1}\left(\hat{\Gamma}_{h}\right)$ \\
\hline (ENP) & $\mathrm{a}_{\mathrm{W}, \Gamma}^{\mathrm{k}}$ & $\mathrm{b}_{\overline{\mathrm{V}}, \Gamma}$ & $\widetilde{H}^{1 / 2}(\Gamma)$ & $H^{-1 / 2}(\Gamma)$ & $\mathcal{S}_{0}^{0,1}\left(\Gamma_{h}\right)$ & $\mathcal{S}_{*}^{-1,0}\left(\hat{\Gamma}_{h}\right)$ \\
\hline
\end{tabular}

From the countless options we examine a particular one applicable to flat screens $\Gamma \subset \mathbb{R}^{2} \times\{0\}$ that allow polar angle parametrization of their boundary, i.e. boundaries that can be described by a function $a(\theta), \theta \in[0,2 \pi]$. In this case, we have tried

$$
S_{\Gamma}(\mathbf{x}, \mathbf{y})=\tan ^{-1}\left(\frac{\sqrt{a\left(\theta_{x}\right)^{2}-r_{x}^{2}} \sqrt{a\left(\theta_{y}\right)^{2}-r_{y}^{2}}}{\sqrt{a\left(\theta_{x}\right) a\left(\theta_{y}\right)}\|\mathbf{x}-\mathbf{y}\|}\right), \quad \text { for } \mathbf{x} \neq \mathbf{y}, \mathbf{x}, \mathbf{y} \in \Gamma,
$$

to construct $b_{\overline{\mathrm{V}}, \Gamma}$ and $\mathrm{b}_{\overline{\mathrm{W}}, \Gamma}$.

6. Numerical Experiments. Numerical experiments were implemented employing BETL2 [14] and the required meshes were generated with Gmsh [8] using polygonal approximation of the boundaries. All required Galerkin matrices were assembled with 12 quadrature points and regularizing transformations [22, Chap. 55. For fine meshes, local low-rank compression of the BEM matrices had to be employed. Specifically, BETL2 uses AHMED for ACA matrix compression [1. The ACA parameters used for these experiments were a tolerance of $10^{-5}$ and admissibility $\eta=0.9$.

The measured condition numbers were computed via the ratio between the maximum and minimum eigenvalues using Preconditioned Conjugate Gradient (PCG)

\footnotetext{
${ }^{5}$ Except for the rank-one regularizations (6.1) and (6.2), where BETL2's default number of quadrature points was used, i.e. 7 points for the mass-matrix and 25 for $\left\langle 1, \omega^{-1}\right\rangle_{\mathbb{D}_{1}}$.
} 
with the Lanczos algorithm [9, Ch.9-10]. We use PCG with a tolerance of $10^{-5}$ for the relative residual norm, initial guess equal to zero and, as right-hand side, we considered the functions $g=\mu=1$, which represent a constant potential for (1.2), and a constant surface charge in (1.5). Since a sufficiently precise computation of the eigenvalues usually requires a larger Krylov subspace and hence more PCG iterations, the algorithm continues iterating until the difference between the newly computed condition number and the old one is less or equal to $10^{-4}$ in two consecutive steps. We measure the performance of a preconditioner by considering the obtained condition numbers $\kappa$ and the number of PCG Iterations, denoted by $I t$, required to solve the linear system with the parameters and right-hand side described above on a mesh with $N$ elements.

For each experiment presented, we provide two tables. The ones on the left, entitled (EDP), contain the preconditioning results for $\mathrm{V}$, while the right-hand tables display the results for $\mathrm{W}$ and is labeled as (ENP). In both situations we compare the performance of our preconditioner $\overline{\mathbf{P}}_{h}$ constructed with our modified BIOs with diagonal preconditioning -denoted by $\mathbf{D}_{h}^{-1}$, and opposite-order operator preconditioning $\mathbf{P}_{h}$ arising from using the standard BIOs ( $c f$. Sections 2.2 and 2.3). We use the same notations for both cases. For the sake of clarity, they are detailed in the next paragraphs:

- (EDP): Here our preconditioner is $\overline{\mathbf{P}}_{h}:=\mathbf{T}_{D, h}^{-1} \overline{\mathbf{V}}_{h} \mathbf{T}_{D, h}^{-T}$ and the oppositeorder preconditioner is $\mathbf{P}_{h}:=\mathbf{T}_{D, h}^{-1} \mathbf{V}_{h} \mathbf{T}_{D, h}^{-T}$, with $\mathbf{T}_{D, h}$ the Galerkin matrix of the $L^{2}$-duality product between $\mathcal{S}^{-1,0}\left(\Gamma_{h}\right)$ and $\mathcal{S}^{0,1}\left(\widehat{\Gamma}_{h}\right)$.

- (ENP): In this case, our preconditioner is $\overline{\mathbf{P}}_{h}:=\mathbf{T}_{N, h}^{-1} \overline{\mathbf{W}}_{h} \mathbf{T}_{N, h}^{-T}$ and $\mathbf{P}_{h}:=$ $\mathbf{T}_{N, h}^{-1} \mathbf{W}_{h} \mathbf{T}_{N, h}^{-T}$, with $\mathbf{T}_{N, h}$ the Galerkin matrix of the $L^{2}$-duality product between $\mathcal{S}_{0}^{0,1}\left(\Gamma_{h}\right)$ and $\mathcal{S}_{*}^{-1,0}\left(\widehat{\Gamma}_{h}\right)$.

Notice that the second term in (3.5) can be regarded as a regularizing term. Without it, the expression would map constants to zero and lose the $H^{1 / 2}\left(\mathbb{D}_{1}\right)$-ellipticity of the bilinear form $b_{\bar{W}, 1}$, which is crucial to construct a suitable preconditioner for $V$, as discussed in Section 2, From an implementation point of view, this regularizing term $\overline{\mathbf{P}}_{h}$ has been set to

$$
\alpha_{\overline{\mathrm{W}}}\left\langle u, \omega^{-1}\right\rangle_{\mathbb{D}_{1}}\left\langle v, \omega^{-1}\right\rangle_{\mathbb{D}_{1}}, \quad u, v \in H^{1 / 2}\left(\mathbb{D}_{1}\right),
$$

with $\alpha_{\overline{\mathrm{W}}}=\frac{1}{2 \pi}=1 /\left\langle 1, \omega^{-1}\right\rangle_{\mathbb{D}_{1}}$.

The bilinear form arising from $\mathrm{W}$ also needs this kind of regularization in order to construct $\mathbf{P}_{h}$ as it maps constants to zero. In the same way as for $\overline{\mathbf{P}}_{h}$, we recover the $H^{1 / 2}\left(\mathbb{D}_{1}\right)$-ellipticity by adding a regularization term [11, Sect. 5.1] equal to (6.1).

6.1. Unit Disk. Tables 6.1.1 and 6.1.2 show the preconditioning results over a disk with two different families of triangular meshes: quasi-uniform and non-quasiuniform (see Figure6.1afor the latter). In both tables, we observe that the condition numbers achieved by $\overline{\mathbf{P}}_{h}$ hardly increase with respect to the number of elements and are asymptotically smaller than those of $\mathbf{P}_{h}$. Nevertheless, the gain in terms of number of PCG iterations is not significant.

A third triangular mesh of the unit disk is also studied. This time, each mesh is generated with local refinement on the boundary such that the meshwidth on $\partial \mathbb{D}_{1}$ is half the one of the previous mesh. This locally refined mesh is displayed in Figure6.1b and the obtained results are reported in Table6.1.3. The condition numbers are just as expected. Moreover, this time we can see that our preconditioner $\overline{\mathbf{P}}_{h}$ not only achieves 
an almost constant $\kappa$, but also its number of PCG iterations becomes significantly smaller than those for $\mathbf{P}_{h}$.

Table 6.1.1: Results on the unit disk with quasi-uniform family of triangular meshes.

(a) (EDP)

\begin{tabular}{|c|cc|cc|cc|}
\hline \multirow{2}{*}{$\mathrm{N}$} & \multicolumn{2}{|c|}{$\mathbf{D}_{h}^{-1} \mathbf{V}_{h}$} & \multicolumn{2}{|c|}{$\mathbf{P}_{h} \mathbf{V}_{h}$} & \multicolumn{2}{|c|}{$\overline{\mathbf{P}}_{h} \mathbf{V}_{h}$} \\
\cline { 2 - 7 } & $\kappa$ & It & $\kappa$ & It & $\kappa$ & It \\
\hline 64 & 22.55 & 11 & 2.71 & 5 & 2.24 & 6 \\
256 & 49.41 & 16 & 2.83 & 6 & 2.48 & 6 \\
1024 & 102.28 & 21 & 3.22 & 6 & 2.63 & 6 \\
4096 & 206.80 & 27 & 3.85 & 7 & 2.72 & 6 \\
16384 & 413.96 & 38 & 4.68 & 8 & 2.87 & 6 \\
\hline
\end{tabular}

(b) (ENP)

\begin{tabular}{|c|cc|cc|cc|}
\hline \multirow{2}{*}{$\mathrm{N}$} & \multicolumn{2}{|c|}{$\mathbf{D}_{h}^{-1} \mathbf{W}_{h}$} & \multicolumn{2}{|c|}{$\mathbf{P}_{h} \mathbf{W}_{h}$} & \multicolumn{2}{|c|}{$\overline{\mathbf{P}}_{h} \mathbf{W}_{h}$} \\
\cline { 2 - 7 } & $\kappa$ & It & $\kappa$ & It & $\kappa$ & It \\
\hline 64 & 2.27 & 4 & 2.09 & 4 & 1.58 & 4 \\
256 & 4.62 & 7 & 2.22 & 5 & 1.40 & 4 \\
1024 & 9.48 & 11 & 2.61 & 6 & 1.38 & 4 \\
4096 & 19.45 & 17 & 3.03 & 7 & 1.38 & 4 \\
16384 & 39.27 & 27 & 3.82 & 8 & 1.51 & 5 \\
\hline
\end{tabular}

Table 6.1.2: Results on the unit disk with non-quasi-uniform family of triangular meshes (see Figure6.1a).

(a) (EDP)

\begin{tabular}{|c|cc|cc|cc|}
\hline \multirow{2}{*}{$\mathrm{N}$} & \multicolumn{2}{|c|}{$\mathbf{D}_{h}^{-1} \mathbf{V}_{h}$} & \multicolumn{2}{|c|}{$\mathbf{P}_{h} \mathbf{V}_{h}$} & \multicolumn{2}{|c|}{$\overline{\mathbf{P}}_{h} \mathbf{V}_{h}$} \\
\cline { 2 - 7 } & $\kappa$ & It & $\kappa$ & It & $\kappa$ & It \\
\hline 96 & 27.27 & 9 & 3.21 & 6 & 3.24 & 6 \\
384 & 55.55 & 14 & 3.41 & 7 & 3.69 & 7 \\
1536 & 110.82 & 21 & 4.14 & 8 & 3.82 & 7 \\
6144 & 230.08 & 27 & 5.01 & 8 & 4.07 & 7 \\
\hline
\end{tabular}

(b) (ENP)

\begin{tabular}{|c|cc|cc|cc|}
\hline \multirow{2}{*}{$\mathrm{N}$} & \multicolumn{2}{|c|}{$\mathbf{D}_{h}^{-1} \mathbf{W}_{h}$} & \multicolumn{2}{|c|}{$\mathbf{P}_{h} \mathbf{W}_{h}$} & \multicolumn{2}{|c|}{$\overline{\mathbf{P}}_{h} \mathbf{W}_{h}$} \\
\cline { 2 - 7 } & $\kappa$ & $\mathrm{It}$ & $\kappa$ & $\mathrm{It}$ & $\kappa$ & $\mathrm{It}$ \\
\hline 96 & 1.95 & 4 & 1.93 & 3 & 1.31 & 3 \\
384 & 4.18 & 6 & 2.47 & 5 & 1.38 & 4 \\
1536 & 8.99 & 10 & 2.92 & 5 & 1.38 & 4 \\
6144 & 19.02 & 16 & 3.39 & 6 & 1.46 & 4 \\
\hline
\end{tabular}

Table 6.1.3: Results on the unit disk with locally refined family of triangular meshes (see Figure 6.1b).

(a) (EDP)

\begin{tabular}{|c|c|cc|cc|cc|}
\hline \multirow{2}{*}{$\mathrm{N}$} & \multirow{2}{*}{$h_{\min }$} & \multicolumn{2}{|c|}{$\mathbf{D}_{h}^{-1} \mathbf{V}_{h}$} & \multicolumn{2}{|c|}{$\mathbf{P}_{h} \mathbf{V}_{h}$} & \multicolumn{2}{c|}{$\overline{\mathbf{P}}_{h} \mathbf{V}_{h}$} \\
\cline { 3 - 8 } & & $\kappa$ & $\mathrm{It}$ & $\kappa$ & $\mathrm{It}$ & $\kappa$ & $\mathrm{It}$ \\
\hline 162 & 0.0568 & 33.09 & 13 & 4.06 & 7 & 4.09 & 8 \\
506 & 0.0244 & 51.23 & 18 & 4.11 & 8 & 4.02 & 8 \\
1052 & 0.0124 & 64.43 & 20 & 4.70 & 8 & 4.00 & 8 \\
2150 & 0.0059 & 79.70 & 20 & 5.59 & 9 & 4.04 & 8 \\
4260 & 0.0030 & 96.09 & 23 & 6.83 & 9 & 4.37 & 9 \\
8398 & 0.0015 & 113.61 & 23 & 7.92 & 10 & 4.53 & 8 \\
16546 & 0.0008 & 131.09 & 25 & 9.00 & 11 & 4.46 & 8 \\
\hline
\end{tabular}

(b) (ENP)

\begin{tabular}{|c|cc|cc|cc|}
\hline \multirow{2}{*}{$\mathrm{N}$} & \multicolumn{2}{|c|}{$\mathbf{D}_{h}^{-1} \mathbf{W}_{h}$} & \multicolumn{2}{|c|}{$\mathbf{P}_{h} \mathbf{W}_{h}$} & \multicolumn{2}{c|}{$\overline{\mathbf{P}}_{h} \mathbf{W}_{h}$} \\
\cline { 2 - 7 } & $\kappa$ & It & $\kappa$ & It & $\kappa$ & It \\
\hline 162 & 2.24 & 5 & 2.17 & 5 & 1.34 & 4 \\
506 & 2.84 & 6 & 3.12 & 6 & 1.69 & 5 \\
1052 & 3.20 & 7 & 3.31 & 6 & 1.52 & 4 \\
2150 & 3.56 & 7 & 3.86 & 7 & 1.58 & 5 \\
4260 & 3.79 & 8 & 4.49 & 8 & 1.61 & 5 \\
8398 & 4.00 & 8 & 5.07 & 8 & 1.62 & 5 \\
16546 & 4.23 & 8 & 5.70 & 9 & 1.64 & 5 \\
\hline
\end{tabular}

Table 6.1.4: Computed best lower bounds for constant $c_{0}$ in Assumption 4.1 (4.6)

\begin{tabular}{|c|cccc|}
\hline \multicolumn{5}{|c|}{ Non-quasi-uniform } \\
\hline $\mathrm{N}$ & 96 & 384 & 1536 & 6144 \\
$c_{0}$ & 3.97 & 4.20 & 4.19 & 4.19 \\
\hline
\end{tabular}

\begin{tabular}{|c|ccccccc|}
\hline \multicolumn{7}{|c|}{ Locally-refined } \\
\hline $\mathrm{N}$ & 162 & 506 & 1052 & 2150 & 4260 & 8398 & 16546 \\
$c_{0}$ & 3.91 & 3.92 & 3.88 & 3.88 & 3.86 & 3.82 & 3.78 \\
\hline
\end{tabular}

6.2. Unit Disk Based Preconditioner for Mapped Screens. In this section we study the preconditioning results achieved when applying the approach described in Section 5. This means that $\mathbf{V}_{h}$ (resp. $\mathbf{W}_{h}$ ) corresponds to the Galerkin matrix of the weakly singular (resp. hypersingular) operator mapped from the disk $\mathbb{D}_{1}$ to the target screen $\Gamma$ via $\phi, \overline{\mathbf{P}}_{h}$ is constructed on the disk, i.e. using (3.8b) (resp. (3.8a)), whereas 


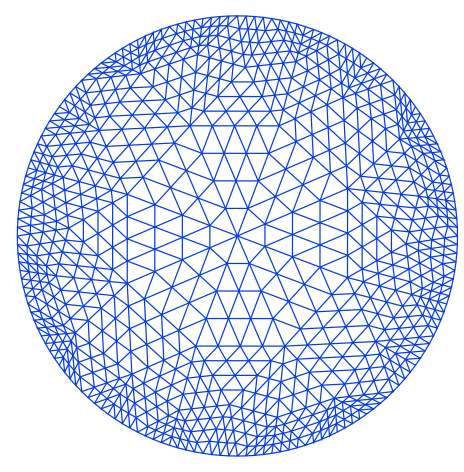

(a) Non-quasi-uniform: The coarsest mesh was created using the functions Attraction and Threshold sequentially in Gmsh. This means the mesh size is a piecewise linear function of the distance to the disk's boundary. The subsequent meshes were obtained by standard refinement in Gmsh.

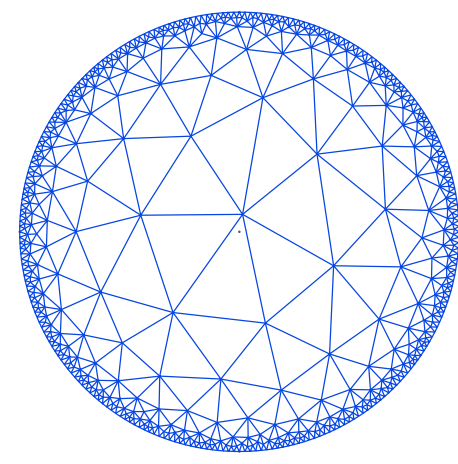

(b) Locally-refined: Each mesh was constructed with the functions Attraction and Matheval in Gmsh, where the evaluated function was the continuous distance to the boundary of the disk plus a parameter $h_{*}>0$. The subsequent meshes were obtained by halving $h_{*}$ and thus the minimum meshwidth on the boundary.

Fig. 6.1: Non-uniform triangular meshes

$\mathbf{P}_{h}$ comes from the standard hypersingular (weakly singular) operator mapped from $\mathbb{D}_{1}$ to $\Gamma$. For this particular case, instead of using (6.1), we regularize $W$ by adding

$$
\alpha_{\mathrm{W}}\langle u, 1\rangle_{\Gamma}\langle v, 1\rangle_{\Gamma}, \quad u, v \in \widetilde{H}^{1 / 2}(\Gamma),
$$

with $\alpha_{\mathrm{W}}=0.3 \approx \frac{1}{\pi}=\left(\langle 1,1\rangle_{\mathbb{D}_{1}}\right)^{-1}$.

We show numerical results for four different shapes: The first one is exhibited in Table 6.2.1 and corresponds to the square screen introduced in Example 1 in Section 5.1. The other three are inspired on Example 2 for different functions $f$ and can be seen in Tables 6.2.2 6.2.4 For all studied mapped screens we compared the performance of the preconditioners on three families of triangular meshes: quasi-uniform, non-quasi-uniform (see Figure 6.1a), and locally refined (see Figure 6.1b).

For the square screen, we observe in Table 6.2.1 the desired optimality of our preconditioner, as the change in $\kappa$ becomes smaller when increasing the number of elements in our meshes. We believe this reflects a pre-asymptotic phase together with numerical errors introduced by quadrature and the ACA approximation. Nevertheless, our preconditioner achieves smaller condition numbers than the other two approaches, particularly on the locally refined mesh. In terms of PCG iterations, we only observe that $\overline{\mathbf{P}}_{h}$ performs significant better than $\mathbf{P}_{h}$ when preconditioning $\mathbf{V}_{h}$.

Tables 6.2.2, 6.2.3 and 6.2.4 reveal the preconditioning results for the other three different shapes mapped from the unit disk. In all of them, we see how the oppositeorder preconditioner $\mathbf{P}_{h}$ displays the expected logarithmic growth while our proposal shows a small rise that seems to be less pronounced the larger the mesh. Naturally, this behaviour is also reflected in the number of PCG iterations. Whereas the number of PCG counts for $\mathbf{P}_{h}$ increases with respect to $N$, those of $\overline{\mathbf{P}}_{h}$ remain constant in the last two levels for most considered mappings. 
Table 6.2.1: Results on mapped square (see formula in (5.1)).

(a) (EDP)

\begin{tabular}{|c|cc|cc|cc|}
\hline \multirow{2}{*}{$\mathrm{N}$} & $\mathbf{D}_{h}^{-1} \mathbf{V}_{h}$ & $\mathbf{P}_{h} \mathbf{V}_{h}$ & \multicolumn{2}{c|}{$\overline{\mathbf{P}}_{h} \mathbf{V}_{h}$} \\
\cline { 2 - 7 } & $\kappa$ & It & $\kappa$ & It & $\kappa$ & It \\
\hline \multicolumn{7}{|c|}{ Quasi-uniform } \\
\hline 64 & 24.08 & 11 & 5.17 & 8 & 2.53 & 7 \\
256 & 51.13 & 17 & 6.06 & 8 & 3.03 & 7 \\
1024 & 105.18 & 22 & 7.10 & 9 & 3.38 & 7 \\
4096 & 217.04 & 30 & 8.33 & 10 & 3.68 & 7 \\
16384 & 441.72 & 39 & 9.90 & 12 & 4.08 & 8 \\
\hline \multicolumn{7}{|c|}{ Non-quasi-uniform } \\
\hline 96 & 27.81 & 10 & 6.77 & 7 & 3.09 & 7 \\
384 & 57.32 & 17 & 7.14 & 9 & 3.81 & 8 \\
1536 & 131.42 & 23 & 9.22 & 10 & 4.39 & 8 \\
6144 & 291.62 & 31 & 10.97 & 11 & 4.72 & 9 \\
\hline \multicolumn{7}{|c|}{ Locally-refined } \\
\hline 162 & 34.05 & 14 & 8.56 & 10 & 4.42 & 9 \\
506 & 52.40 & 18 & 9.19 & 11 & 4.39 & 9 \\
1052 & 67.15 & 20 & 9.94 & 10 & 4.35 & 9 \\
2150 & 82.39 & 24 & 11.99 & 13 & 4.59 & 8 \\
4260 & 100.78 & 25 & 14.12 & 13 & 4.94 & 9 \\
8398 & 117.02 & 28 & 16.21 & 14 & 5.17 & 9 \\
16546 & 132.73 & 30 & 18.68 & 15 & 5.02 & 9 \\
\hline
\end{tabular}

(b) (ENP)

\begin{tabular}{|c|cc|cc|cc|}
\hline \multirow{2}{*}{$\mathrm{N}$} & $\mathbf{D}_{h}^{-1} \mathbf{W}_{h}$ & \multicolumn{2}{|c|}{$\mathbf{P}_{h} \mathbf{W}_{h}$} & \multicolumn{2}{|c|}{$\overline{\mathbf{P}}_{h} \mathbf{W}_{h}$} \\
\cline { 2 - 7 } & $\kappa$ & It & $\kappa$ & It & $\kappa$ & It \\
\hline \multicolumn{7}{|c|}{ Quasi-uniform } \\
\hline 64 & 2.26 & 4 & 2.38 & 5 & 1.80 & 5 \\
256 & 4.85 & 8 & 2.55 & 6 & 2.00 & 6 \\
1024 & 10.16 & 12 & 3.02 & 6 & 2.32 & 7 \\
4096 & 21.40 & 18 & 3.51 & 7 & 2.57 & 8 \\
16384 & 44.03 & 29 & 4.06 & 8 & 2.77 & 8 \\
\hline \multicolumn{7}{|c|}{ Non-quasi-uniform } \\
\hline 96 & 1.99 & 4 & 2.16 & 4 & 1.63 & 5 \\
384 & 4.38 & 6 & 2.78 & 5 & 2.17 & 7 \\
1536 & 10.07 & 11 & 3.29 & 6 & 2.54 & 7 \\
6144 & 21.44 & 17 & 3.80 & 7 & 2.78 & 8 \\
\hline \multicolumn{7}{|c|}{ Locally-refined } \\
\hline 162 & 2.36 & 5 & 2.37 & 5 & 1.88 & 6 \\
506 & 3.06 & 7 & 3.49 & 7 & 2.49 & 7 \\
1052 & 3.56 & 8 & 3.69 & 7 & 2.65 & 7 \\
2150 & 3.87 & 8 & 4.25 & 7 & 2.58 & 7 \\
4260 & 4.18 & 9 & 4.82 & 8 & 2.81 & 7 \\
8398 & 4.49 & 9 & 5.54 & 9 & 2.78 & 7 \\
16546 & 4.78 & 9 & 6.12 & 10 & 2.85 & 7 \\
\hline
\end{tabular}

Table 6.2.2: Results on mapped screens $\boldsymbol{\phi}(\mathbf{x})=\left(x_{1}, x_{2}, x_{1}+x_{2}\right)^{T}$.

(a) (EDP)

\begin{tabular}{|c|c|c|c|c|c|c|}
\hline \multirow[t]{2}{*}{$\mathrm{N}$} & \multicolumn{2}{|c|}{$\mathbf{D}_{h}^{-1} \mathbf{V}_{h}$} & \multicolumn{2}{|c|}{$\mathbf{P}_{h} \mathbf{V}_{h}$} & \multicolumn{2}{|c|}{$\overline{\mathbf{P}}_{h} \mathbf{V}_{h}$} \\
\hline & $\kappa$ & It & $\kappa$ & It & $\kappa$ & It \\
\hline \multicolumn{7}{|c|}{ Quasi-uniform } \\
\hline 64 & 24.15 & 11 & 3.44 & 7 & 3.02 & 7 \\
\hline 256 & 51.65 & 17 & 4.17 & 8 & 3.28 & 7 \\
\hline 1024 & 108.97 & 22 & 4.58 & 8 & 3.45 & 7 \\
\hline 4096 & 223.37 & 31 & 5.76 & 9 & 3.61 & 7 \\
\hline 16384 & 435.05 & 40 & 6.70 & 10 & 3.72 & 7 \\
\hline \multicolumn{7}{|c|}{ Non-quasi-uniform } \\
\hline 96 & 27.42 & 11 & 3.88 & 7 & 3.93 & 8 \\
\hline 384 & 55.80 & 17 & 4.28 & 8 & 4.35 & 8 \\
\hline 1536 & 116.76 & 22 & 5.49 & 9 & 4.69 & 8 \\
\hline 6144 & 249.66 & 29 & 6.56 & 10 & 4.91 & 8 \\
\hline \multicolumn{7}{|c|}{ Locally-refined } \\
\hline 162 & 33.01 & 13 & 5.01 & 9 & 4.67 & 9 \\
\hline 506 & 51.70 & 16 & 6.04 & 9 & 5.14 & 9 \\
\hline 1052 & 65.04 & 19 & 6.79 & 10 & 5.09 & 9 \\
\hline 2150 & 80.34 & 22 & 7.93 & 10 & 5.30 & 9 \\
\hline 4260 & 97.31 & 23 & 9.17 & 11 & 5.62 & 10 \\
\hline 8398 & 114.45 & 24 & 10.42 & 12 & 5.97 & 9 \\
\hline 16546 & 133.70 & 27 & 11.79 & 12 & 5.76 & 9 \\
\hline
\end{tabular}

(b) (ENP)

\begin{tabular}{|c|cc|cc|cc|}
\hline \multirow{2}{*}{$\mathrm{N}$} & $\mathbf{D}_{h}^{-1} \mathbf{W}_{h}$ & $\mathbf{P}_{h} \mathbf{W}_{h}$ & \multicolumn{2}{|c|}{$\overline{\mathbf{P}}_{h} \mathbf{W}_{h}$} \\
\cline { 2 - 7 } & $\kappa$ & It & $\kappa$ & It & $\kappa$ & It \\
\hline \multicolumn{7}{|c|}{ Quasi-uniform } \\
\hline 64 & 2.69 & 6 & 2.16 & 5 & 1.92 & 5 \\
256 & 5.38 & 8 & 2.30 & 5 & 1.95 & 6 \\
1024 & 11.04 & 13 & 2.72 & 6 & 2.06 & 6 \\
4096 & 22.67 & 19 & 3.15 & 7 & 2.12 & 6 \\
16384 & 43.65 & 31 & 3.67 & 8 & 2.14 & 6 \\
\hline \multicolumn{7}{|c|}{ Non-quasi-uniform } \\
\hline 96 & 2.21 & 5 & 2.00 & 4 & 1.69 & 5 \\
384 & 4.69 & 8 & 2.56 & 5 & 1.92 & 6 \\
1536 & 9.70 & 12 & 3.03 & 6 & 2.05 & 6 \\
6144 & 20.32 & 18 & 3.52 & 7 & 2.14 & 6 \\
\hline \multicolumn{7}{|c|}{ Locally-refined } \\
\hline 162 & 2.43 & 6 & 2.24 & 5 & 1.75 & 5 \\
506 & 3.33 & 8 & 3.27 & 7 & 2.03 & 6 \\
1052 & 3.84 & 9 & 3.43 & 7 & 2.00 & 6 \\
2150 & 4.23 & 9 & 4.04 & 7 & 2.10 & 6 \\
4260 & 4.77 & 10 & 4.68 & 8 & 2.17 & 6 \\
8398 & 4.96 & 10 & 5.23 & 8 & 2.24 & 6 \\
16546 & 5.48 & 11 & 5.91 & 9 & 2.31 & 6 \\
\hline
\end{tabular}

Furthermore, we can see that our proposed preconditioner performs considerably better for locally refined meshes when the distortion of the mesh is moderate, as in Tables 6.2 .2 and 6.2 .3 where $f\left(x_{1}, x_{2}\right)=x_{1}+x_{2}$ and $f\left(x_{1}, x_{2}\right)=x_{1} x_{2}$, respectively. 
However, as predicted in Remark 4, this is not the case for $f\left(x_{1}, x_{2}\right)=x_{1}^{2}+x_{2}^{2}$. In that case, $\overline{\mathbf{P}}_{h}$ does not actually offer an advantage over $\mathbf{P}_{h}$ in terms of PCG counts, in spite of achieving an almost constant condition number.

Table 6.2.3: Results on mapped screens $\phi(\mathbf{x})=\left(x_{1}, x_{2}, x_{1} x_{2}\right)^{T}$.

(a) (EDP)

\begin{tabular}{|c|cc|cc|cc|}
\hline \multirow{2}{*}{$\mathrm{N}$} & $\mathbf{D}_{h}^{-1} \mathbf{V}_{h}$ & \multicolumn{2}{|c|}{$\mathbf{P}_{h} \mathbf{V}_{h}$} & \multicolumn{2}{|c|}{$\overline{\mathbf{P}}_{h} \mathbf{V}_{h}$} \\
\cline { 2 - 7 } & $\kappa$ & It & $\kappa$ & It & $\kappa$ & It \\
\hline \multicolumn{7}{|c|}{ Quasi-uniform } \\
\hline 64 & 24.29 & 11 & 3.11 & 6 & 2.38 & 5 \\
256 & 51.36 & 16 & 3.68 & 7 & 2.56 & 6 \\
1024 & 104.49 & 21 & 4.36 & 7 & 2.77 & 6 \\
4096 & 211.30 & 28 & 5.16 & 8 & 2.95 & 7 \\
16384 & 423.24 & 37 & 6.13 & 9 & 3.09 & 7 \\
\hline \multicolumn{7}{|c|}{ Non-quasi-uniform } \\
\hline 96 & 27.87 & 8 & 4.70 & 6 & 2.69 & 6 \\
384 & 56.90 & 15 & 5.04 & 7 & 3.33 & 7 \\
1536 & 113.59 & 20 & 5.86 & 8 & 3.82 & 8 \\
6144 & 233.21 & 25 & 6.74 & 9 & 4.05 & 8 \\
\hline \multicolumn{7}{|c|}{ Locally-refined } \\
\hline 162 & 33.89 & 12 & 6.22 & 8 & 4.08 & 8 \\
506 & 52.87 & 15 & 5.95 & 8 & 4.25 & 9 \\
1052 & 66.45 & 19 & 6.57 & 9 & 4.14 & 9 \\
2150 & 82.45 & 20 & 7.93 & 9 & 4.57 & 9 \\
4260 & 99.14 & 23 & 9.45 & 11 & 4.90 & 9 \\
8398 & 117.08 & 25 & 10.92 & 11 & 5.25 & 9 \\
16546 & 135.73 & 27 & 12.64 & 12 & 5.10 & 9 \\
\hline
\end{tabular}

(b) (ENP)

\begin{tabular}{|c|cc|cc|cc|}
\hline \multirow{2}{*}{$\mathrm{N}$} & $\mathbf{D}_{h}^{-1} \mathbf{W}_{h}$ & $\mathbf{P}_{h} \mathbf{W}_{h}$ & \multicolumn{2}{|c|}{$\overline{\mathbf{P}}_{h} \mathbf{W}_{h}$} \\
\cline { 2 - 7 } & $\kappa$ & It & $\kappa$ & It & $\kappa$ & It \\
\hline \multicolumn{7}{|c|}{ Quasi-uniform } \\
\hline 64 & 2.28 & 5 & 2.14 & 4 & 1.64 & 4 \\
256 & 4.65 & 7 & 2.30 & 5 & 1.56 & 5 \\
1024 & 9.65 & 11 & 2.71 & 6 & 1.60 & 5 \\
4096 & 19.99 & 17 & 3.14 & 7 & 1.65 & 5 \\
16384 & 40.31 & 27 & 3.63 & 8 & 1.68 & 6 \\
\hline \multicolumn{7}{|c|}{ Non-quasi-uniform } \\
\hline 96 & 1.94 & 4 & 2.01 & 3 & 1.40 & 4 \\
384 & 4.19 & 6 & 2.57 & 5 & 1.66 & 5 \\
1536 & 9.14 & 10 & 3.04 & 5 & 1.75 & 5 \\
6144 & 19.35 & 16 & 3.52 & 6 & 1.82 & 5 \\
\hline \multicolumn{7}{|c|}{ Locally-refined } \\
\hline 162 & 2.30 & 5 & 2.25 & 5 & 1.50 & 4 \\
506 & 3.03 & 7 & 3.24 & 6 & 1.83 & 5 \\
1052 & 3.35 & 8 & 3.46 & 6 & 1.70 & 5 \\
2150 & 3.82 & 8 & 4.02 & 7 & 1.82 & 5 \\
4260 & 4.08 & 9 & 4.68 & 8 & 1.88 & 5 \\
8398 & 4.40 & 9 & 5.29 & 8 & 1.88 & 5 \\
16546 & 4.76 & 10 & 5.96 & 9 & 1.92 & 6 \\
\hline
\end{tabular}

Table 6.2.4: Results on mapped screens $\phi(\mathbf{x})=\left(x_{1}, x_{2}, x_{1}^{2}+x_{2}^{2}\right)^{T}$.

(a) (EDP)

\begin{tabular}{|c|c|c|c|c|c|c|}
\hline \multirow[t]{2}{*}{$\mathrm{N}$} & \multicolumn{2}{|c|}{$\mathbf{D}_{h}^{-1} \mathbf{V}_{h}$} & \multicolumn{2}{|c|}{$\mathbf{P}_{h} \mathbf{V}_{h}$} & \multicolumn{2}{|c|}{$\overline{\mathbf{P}}_{h} \mathbf{V}_{h}$} \\
\hline & $\kappa$ & It & $\kappa$ & It & $\kappa$ & It \\
\hline \multicolumn{7}{|c|}{ Quasi-uniform } \\
\hline 64 & 25.97 & 10 & 1.88 & 5 & 4.25 & 7 \\
\hline 256 & 54.86 & 15 & 2.20 & 6 & 4.83 & 9 \\
\hline 1024 & 111.73 & 19 & 2.57 & 6 & 5.21 & 10 \\
\hline 4096 & 228.14 & 25 & 3.05 & 7 & 5.47 & 10 \\
\hline 16384 & 462.53 & 33 & 3.69 & 8 & 5.66 & 11 \\
\hline \multicolumn{7}{|c|}{ Non-quasi-uniform } \\
\hline 96 & 31.20 & 9 & 3.00 & 6 & 3.35 & 7 \\
\hline 384 & 63.71 & 14 & 3.44 & 7 & 5.25 & 8 \\
\hline 1536 & 126.36 & 18 & 3.85 & 7 & 5.91 & 10 \\
\hline 6144 & 253.70 & 23 & 4.22 & 8 & 6.18 & 10 \\
\hline \multicolumn{7}{|c|}{ Locally-refined } \\
\hline 162 & 39.59 & 14 & 4.11 & 7 & 4.78 & 9 \\
\hline 506 & 63.88 & 18 & 3.39 & 7 & 5.14 & 10 \\
\hline 1052 & 82.96 & 21 & 3.84 & 7 & 5.24 & 10 \\
\hline 2150 & 102.92 & 23 & 4.66 & 8 & 5.69 & 11 \\
\hline 4260 & 125.85 & 27 & 5.70 & 8 & 6.13 & 11 \\
\hline 8398 & 150.11 & 29 & 6.67 & 9 & 6.49 & 12 \\
\hline 16546 & 175.90 & 32 & 7.71 & 10 & 6.54 & 12 \\
\hline
\end{tabular}

(b) (ENP)

\begin{tabular}{|c|cc|cc|cc|}
\hline \multirow{2}{*}{$\mathrm{N}$} & $\mathbf{D}_{h}^{-1} \mathbf{W}_{h}$ & \multicolumn{2}{|c|}{$\mathbf{P}_{h} \mathbf{W}_{h}$} & $\overline{\mathbf{P}}_{h} \mathbf{W}_{h}$ \\
\cline { 2 - 7 } & $\kappa$ & It & $\kappa$ & It & $\kappa$ & It \\
\hline \multicolumn{7}{|c|}{ Quasi-uniform } \\
\hline 64 & 3.91 & 6 & 2.26 & 5 & 2.98 & 6 \\
256 & 8.39 & 9 & 2.10 & 5 & 3.13 & 7 \\
1024 & 17.48 & 14 & 2.47 & 6 & 3.29 & 8 \\
4096 & 36.31 & 21 & 2.89 & 6 & 3.41 & 8 \\
16384 & 73.67 & 32 & 3.38 & 7 & 3.48 & 8 \\
\hline \multicolumn{7}{|c|}{ Non-quasi-uniform } \\
\hline 96 & 3.40 & 5 & 1.95 & 4 & 2.64 & 5 \\
384 & 7.80 & 8 & 2.39 & 5 & 3.28 & 7 \\
1536 & 16.25 & 12 & 2.80 & 5 & 3.46 & 8 \\
6144 & 34.19 & 18 & 3.28 & 6 & 3.59 & 8 \\
\hline \multicolumn{7}{|c|}{ Locally-refined } \\
\hline 162 & 3.96 & 7 & 2.24 & 5 & 2.92 & 6 \\
506 & 5.26 & 9 & 3.58 & 7 & 3.60 & 8 \\
1052 & 5.99 & 10 & 3.71 & 7 & 3.59 & 8 \\
2150 & 6.64 & 11 & 4.37 & 8 & 3.76 & 8 \\
4260 & 7.09 & 11 & 5.23 & 9 & 3.86 & 8 \\
8398 & 7.43 & 12 & 6.02 & 9 & 3.97 & 8 \\
16546 & 7.85 & 12 & 6.54 & 10 & 3.94 & 8 \\
\hline \multicolumn{7}{|c|}{}
\end{tabular}


6.3. Shape-intrinsic preconditioners for flat screens. We lack theoretical predictions for the performance of the construction (5.12) from Section 5.3. We can, however, report numerical results for the unit square $\Gamma:=(0,1) \times\{0\}$ and two families of triangular meshes: quasi-uniform and non-uniform (see Figure 6.2 for a specimen of each type).

Table 6.3.1 gives the corresponding numerical results for preconditioning on a unit square screen. In Case (EDP), we used $\alpha_{\mathrm{W}}=\alpha_{\overline{\mathrm{W}}}=0.3$ for $\mathbf{P}_{h}$ and $\overline{\mathbf{P}}_{h}$, respectively. We observe that the shape-intrinsic preconditioners behave qualitatively in the same fashion as the unit disk based preconditioner for mapped screens presented in Section 5. taking about two iterations less to reach the desired tolerance in most cases.

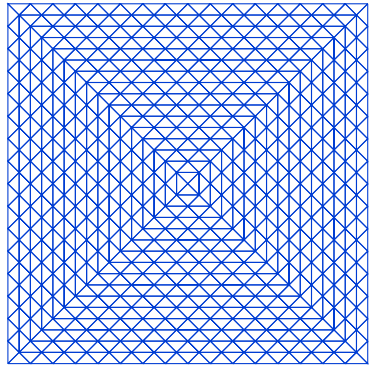

(a) Quasi-uniform

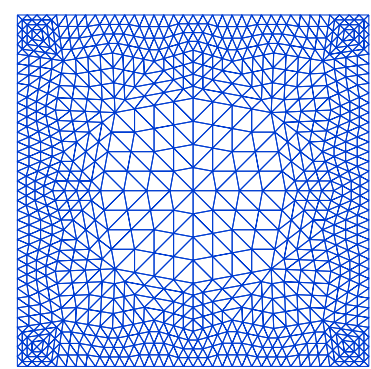

(b) Non-uniform

Fig. 6.2: Triangular meshes for unit square

Table 6.3.1: Results for a square screen. $\overline{\mathbf{P}}_{h}$ built using shape-intrinsic preconditioner for flat screens.

(a) (EDP)

\begin{tabular}{|c|cc|cc|cc|}
\hline \multirow{2}{*}{$\mathrm{N}$} & $\mathbf{D}_{h}^{-1} \mathbf{V}_{h}$ & \multicolumn{2}{|c|}{$\mathbf{P}_{h} \mathbf{V}_{h}$} & \multicolumn{2}{|c|}{$\overline{\mathbf{P}}_{h} \mathbf{V}_{h}$} \\
\cline { 2 - 7 } & $\kappa$ & It & $\kappa$ & It & $\kappa$ & It \\
\hline \multicolumn{7}{|c|}{ Quasi-uniform } \\
\hline 64 & 22.55 & 8 & 3.15 & 4 & 3.32 & 4 \\
256 & 50.09 & 15 & 4.30 & 6 & 3.70 & 5 \\
1024 & 103.14 & 20 & 5.31 & 6 & 3.94 & 6 \\
4096 & 207.86 & 26 & 6.41 & 9 & 4.10 & 6 \\
16384 & 416.51 & 34 & 7.64 & 9 & 4.22 & 7 \\
\hline \multicolumn{7}{|c|}{ Non-uniform } \\
\hline 108 & 24.98 & 9 & 3.64 & 6 & 3.87 & 7 \\
432 & 56.88 & 15 & 4.50 & 8 & 4.58 & 8 \\
1728 & 118.19 & 21 & 5.78 & 8 & 5.15 & 8 \\
6912 & 241.51 & 27 & 6.94 & 9 & 5.58 & 9 \\
27648 & 481.86 & 38 & 8.19 & 10 & 5.92 & 9 \\
\hline
\end{tabular}

(b) (ENP)

\begin{tabular}{|c|cc|cc|cc|}
\hline \multirow{2}{*}{$\mathrm{N}$} & $\mathbf{D}_{h}^{-1} \mathbf{W}_{h}$ & \multicolumn{2}{|c|}{$\mathbf{P}_{h} \mathbf{W}_{h}$} & \multicolumn{2}{|c|}{$\overline{\mathbf{P}}_{h} \mathbf{W}_{h}$} \\
\cline { 2 - 7 } & $\kappa$ & It & $\kappa$ & It & $\kappa$ & It \\
\hline \multicolumn{7}{|c|}{ Quasi-uniform } \\
\hline 64 & 2.46 & 5 & 2.02 & 4 & 1.41 & 4 \\
256 & 4.84 & 7 & 2.44 & 5 & 1.44 & 4 \\
1024 & 9.76 & 10 & 2.87 & 5 & 1.45 & 5 \\
4096 & 19.43 & 18 & 4.19 & 8 & 1.83 & 6 \\
16384 & 41.79 & 30 & 7.69 & 12 & 3.41 & 10 \\
\hline \multicolumn{8}{|c|}{ Non-uniform } \\
\hline 108 & 2.05 & 3 & 2.29 & 4 & 1.46 & 4 \\
432 & 4.40 & 7 & 2.64 & 5 & 1.47 & 5 \\
1728 & 9.29 & 11 & 3.08 & 6 & 1.50 & 5 \\
6912 & 19.18 & 16 & 3.69 & 6 & 1.52 & 5 \\
27648 & 39.06 & 24 & 4.70 & 8 & 1.68 & 6 \\
\hline
\end{tabular}

7. Conclusion. We have developed dual mesh-based operator preconditioning for Galerkin BEM on three-dimensional screens using new modified hypersingular and weakly singular integral operators that supply the exact inverses for the weakly singular and hypersingular operators on disks, respectively. We have proved that our 
approach accomplishes $h$-independent condition numbers for parametrized screens, with remarkable success for non-uniform meshes. However, its performance depends on the extent of deformation of the screen $\Gamma$ with respect to the unit disk $\mathbb{D}_{1}$. We observed that the resulting condition numbers may be rather large depending on the transformation $\phi$.

The asymptotic optimality of our new preconditioners has been investigated via exhaustive numerical experiments on both sequences of uniform and locally refined meshes. We have confirmed that our method achieves the expected performance for all studied shapes of screens.

In addition, we briefly presented shape-intrinsic preconditioners that rely on heuristic approximation of the modified BIOs $\bar{V}$ and $\bar{W}$. However, rigorous analysis remains elusive.

Computationally, substantial additional work is required for the modified operators $\bar{V}$ and $\bar{W}$. Yet, these are completely data-local and in-cache and, thus, our C++ code took only marginally longer to assemble the associated matrices compared to building those for the standard boundary integral operators.

We emphasize that this work has a theoretical focus. In applications, "opposite order preconditioning" may be almost as good in terms of number of iterations required. Then the new preconditioners may not be worth the effort, in spite of their asympotic optimality.

Our current work is aimed at extending these preconditioning techniques to Helmholtz and Maxwell equations.

\section{REFERENCES}

[1] M. Bebendorf, Another software library on hierarchical matrices for elliptic differential equations (ahmed), Universität Leipzig, Fakultät für Mathematik und Informatik, (2005).

[2] O. P. Bruno And S. K. Lintner, Second-kind integral solvers for TE and TM problems of diffraction by open arcs, Radio Science, 47 (2012), p. 6006.

[3] O. P. Bruno And S. K. Lintner, A high-order integral solver for scalar problems of diffraction by screens and apertures in three-dimensional space, J. Comput. Phys., 252 (2013), pp. 250274.

[4] A. Buffa And S. Christiansen, A dual finite element complex on the barycentric refinement, Mathematics of Computation, 76 (2007), pp. 1743-1769.

[5] S. H. Christiansen, Résolution des équations intégrales pour la diffraction d'ondes acoustiques et électromagnétiques - Stabilisation d'algorithmes itératifs et aspects de l'analyse numérique, theses, Ecole Polytechnique X, Jan. 2002.

[6] S. H. Christiansen and J.-C. Nédélec, Preconditioners for the boundary element method in acoustics, in Mathematical and numerical aspects of wave propagation (Santiago de Compostela, 2000), SIAM, Philadelphia, PA, 2000, pp. 776-781.

[7] M. Costabel, M. Dauge, and R. Duduchava, Asymptotics without logarithmic terms for crack problems, Comm. Partial Differential Equations, 28 (2003), pp. 869-926.

[8] C. Geuzaine and J.-F. Remacle, Gmsh: A 3-d finite element mesh generator with built-in preand post-processing facilities, International Journal for Numerical Methods in Engineering, 79 (2009), pp. 1309-1331.

[9] G. H. Golub and C. F. Van Loan, Matrix computations, Johns Hopkins Studies in the Mathematical Sciences, Johns Hopkins University Press, Baltimore, MD, third ed., 1996.

[10] R. HiptmaiR, Operator preconditioning, Computers and Mathematics with Applications, 52 (2006), pp. 699-706.

[11] R. Hiptmair, C. Jerez-Hanckes, and C. Urzúa-Torres, Mesh-independent operator preconditioning for boundary elements on open curves., SIAM J. Numerical Analysis, 52 (2014), pp. 2295-2314.

[12] - Optimal operator preconditioning for weakly singular operator over 3d screens, Tech. Rep. 2017-13, Seminar for Applied Mathematics, ETH Zürich, Switzerland, 2017 (Preprint). https://www.sam.math.ethz.ch/sam_reports/reports_final/reports2017/2017-13_fp.pdf 
[13] R. Hiptmair, C. Jerez-Hanckes, and C. Urzúa-Torres, Closed-Form Inverses of the Weakly Singular and Hypersingular Operators on Disks, Integral Equations Operator Theory, 90 (2018), p. 90:4.

[14] R. Hiptmair And L. Kielhorn, BETL - A generic boundary element template library, Tech. Rep. 2012-36, Seminar for Applied Mathematics, ETH Zurich, 2012. https://www.sam.math.ethz.ch/sam_reports/reports_final/reports2012/2012-36.pdf

[15] R. Hiptmair and C. Urzúa-Torres, Dual Mesh Operator Preconditioning On $3 D$ Screens: Low-Order Boundary Element Discretization., Tech. Rep. 2016-14, Seminar for Applied Mathematics, ETH Zürich, Switzerland, 2016 (Preprint). https://www.sam.math.ethz.ch/sam_reports/reports_final/reports2016/2016-14.pdf

[16] C. Jerez-HANCKes AND J. NÉDÉLEC, Explicit variational forms for the inverses of integral logarithmic operators over an interval, SIAM Journal on Mathematical Analysis, 44 (2012), pp. 2666-2694.

[17] J. Lions And F. Magenes, Nonhomogeneous boundary value problems and applications, Springer, Berlin, 1972.

[18] W. MCLeAn, Strongly elliptic systems and boundary integral equations, Cambridge University Press, Cambridge, UK, 2000.

[19] W. McLean And O. Steinbach, Boundary element preconditioners for a hypersingular integral equations on an interval, Adv. Comp. Math., 11 (1999), pp. 271-286.

[20] P. Ramaciotti And J.-C. NédÉlec, About Some Boundary Integral Operators on the Unit Disk Related to the Laplace Equation, SIAM J. Numer. Anal., 55 (2017), pp. 1892-1914.

[21] P. Ramaciotti Morales, Theoretical and numerical aspects of wave propagation phenomena in complex domains and applications to remote sensing, theses, Université Paris-Saclay, Oct. 2016.

[22] S. Sauter and C. Schwab, Boundary element methods, vol. 39 of Springer Series in Computational Mathematics, Springer, Heidelberg, 2010.

[23] O. Steinbach, Stability estimates for hybrid coupled domain decomposition methods, vol. 1809 of Lecture Notes in Mathematics, Springer, Berlin, 2003.

[24] — Numerical approximation methods for elliptic boundary value problems: Finite and boundary elements, Springer, New York, 2008.

[25] O. Steinbach and W. Wendland, The construction of some efficient preconditioners in the boundary element method, Adv. Comput. Math, 9 (1998), pp. 191-216.

[26] E. Stephan, Boundary integral equations for screen problems in $\mathbb{R}^{3}$, Integral Equations and Operator Theory, 10 (1987), pp. 236-257.

[27] E. P. Stephan And W. L. Wendland, An augmented Galerkin procedure for the boundary integral method applied to two-dimensional screen and crack problems, Applicable Anal., 18 (1984), pp. 183-219.

[28] C. URzúA-Torres, Operator Preconditioning for Galerkin Boundary Element Methods on Screens, PhD thesis, ETH Zurich, 2018. 